\title{
Risk-based Automated Assessment and Testing for the Cybersecurity Certification and Labelling of IoT Devices
}

\author{
Sara N. Matheu-García ${ }^{\mathrm{a}, *}$, José L. Hernández-Ramos ${ }^{\mathrm{a}, \mathrm{c}}$, Antonio F. Skarmeta ${ }^{\mathrm{a}, \mathrm{b}}$, Gianmarco Baldinic \\ ${ }^{a}$ Department Information and Communication Engineering (DIIC), Faculty of Computer Science, University of Murcia, Murcia 30100, \\ Spain \\ ${ }^{b}$ Odin Solutions, Murcia, Spain \\ ${ }^{c}$ European Commission, Joint Research Centre, Ispra 21027, Italy
}

\begin{abstract}
Nowadays, security aspects represent one of the most significant barriers for the adoption of largescale Internet of Things (IoT) deployments. In this sense, being able to certify and communicate the security level of a certain device is crucial for their acceptance. Towards this end, we propose a security certification methodology designed for IoT to empower different stakeholders with the ability to assess security solutions for large-scale IoT deployments in an automated way. It also supports transparency on the IoT security level to the consumers because the methodology provides a label as one of the main results of the certification process. The certification approach represents an instantiation of the Risk-based Security Assessment and Testing methodologies presented by ETSI based on the ISO 31000 and ISO 29119, and it is built on top of different technologies and approaches for security testing and risk assessment adapted to the IoT landscape. As a proof of concept, the proposed methodology is applied to one of the scenarios proposed in the scope of the Horizon 2020 ARMOUR project for assessing the fulfilment of several security properties of IoT devices.
\end{abstract}

Keywords: Certification, MBT, TTCN, IoT, Security, Security Testing, Security Risk Assessment, Security Labelling

2010 MSC: 00-01, 99-00

\section{Introduction}

Nowadays, security aspects represent one of the most significant barriers for the adoption of large-scale Internet of Things (IoT) deployments [1] [2]. Recent attacks such as Hajime, IoT Reaper, BrickerBot or Mirai [3], exploit the vulnerabilities of IoT devices to control them and create IoT 5 botnets, which are usually used to perform denial of service (DoS) attacks. As a result of these vulnerabilities, manufacturers of IoT devices are working together with standardization bodies, to build the next generation of more secure and standardized smart objects, but security certification

\footnotetext{
${ }^{*}$ Corresponding author

Email addresses: saranieves .matheu@um. es (Sara N. Matheu-García), jose-luis .hernandez-ramos@ec. europa.eu (José L. Hernández-Ramos), skarmeta@um. es (Antonio F. Skarmeta), gianmarco.baldini@ec . europa.eu (Gianmarco Baldini)
} 
remains a challenging task. Security threats are increasing due to the ubiquitous nature of the evolution of Internet towards IoT, raising major concerns for companies, governments, regulatory bodies and end-users. A suitable security certification scheme [4] would help to assess and compare different security technologies, in order to provide a more harmonized IoT security view to be leveraged by end consumers. To support transparency to the user on the outcome of the certification process, this paper proposes the concept of a cybersecurity label (and a related labelling process), which contains information related with the level of security validated in the certification process.

Indeed, the European Cyber Security Organisation Working Group 1 (ECSO WG1) [5] is working on standardisation, certification, labelling and supply chain management, developing a roadmap for the development of security standards and certification. The European Union Agency for Network and Information Security (ENISA) [4] also discusses the main challenges regarding security and privacy of online seals and proposes solutions, such as a label or icon showing the different dimensions of security, and how the label can be linked to the certification process.

However, a certification methodology for security in IoT must overcome different obstacles that are inherent to the IoT context and the IoT device life cycle. On one hand, the high degree of diversity and heterogeneity of devices and products is in conflict with the need for objective comparisons regarding security aspects. On other hand, due to the dynamism of typical IoT environments (roaming, security and configuration changes, etc.), the certification methodology must take into account these changing conditions, managing the device life cycle and taking into account the context in which the IoT device will be operating. Therefore, agile self-assessment schemes and test automation environments should be created and evolved to ensure products have a minimum security level appropriate for a context where they are used [6]. Towards this end, a clear identification of threats and vulnerabilities is key to guarantee the success of the approach. In addition, the methodology must cope with the business requirements and needs from the IoT market. It means that security certification approaches should be efficient and cost effective, so the product launch in the market is not significantly delayed. Another challenge is how to communicate the result in a way that is understood by the user [4].

To cope with these challenges, this work presents a certification methodology for IoT security that is based on two building blocks: security risk assessment and security testing. This methodology aims to certify the device's security within a specific context. In particular, our approach is based on an instantiation of the security risk assessment and testing methodology proposed by the European Telecommunications Standards Institute (ETSI) [7]. The methodology makes use 40 of tools such as Model-Based Testing (MBT) [8], TITAN ${ }^{1}$ and FIT IoT Lab ${ }^{2}$ to perform the testing in an automated and scalable way, and Common Vulnerability Scoring System (CVSS) [9] for the security risk assessment process. We have also developed a benchmarking tool to automate the integration between security testing and risk assessment, in order to provide a more visual result of the process. In addition, we have applied the proposed methodology to a specific scenario based on widely used IoT protocols, such as the Constrained Application Protocol (CoAP) [10] and Datagram Transport Layer Security (DTLS) [11], in order to prove the feasibility and soundness of our proposal.In a previous work [12], we partially addressed the risk assessment block. However, in this paper, we detail all the insights of the process, we address the testing part, automating the certification process, and we present an integration between the ETSI approach and the tools

\footnotetext{
${ }^{1}$ http://www.tten-3.org/

${ }^{2}$ https:/ / www.iot-lab.info/
} 
Table 1: Glossary of terms

\begin{tabular}{|c|c|c|}
\hline Definition & Description & Source \\
\hline Certification & $\begin{array}{l}\text { A comprehensive assessment to determine the extent to which the controls } \\
\text { are implemented correctly, operating as intended and producing the desired } \\
\text { outcome with respect to meeting the security requirements for the system }\end{array}$ & FIPS $200\lfloor 13$ \\
\hline $\begin{array}{l}\text { Evaluation Assur- } \\
\text { ance Level (EAL) }\end{array}$ & $\begin{array}{l}\text { Set of assurance requirements that represent a point on the Common Criteria } \\
\text { predefined assurance scale }\end{array}$ & $\begin{array}{l}\text { CNSSI-4009 } \\
\text { [14 }\end{array}$ \\
\hline $\begin{array}{l}\text { Information Security } \\
\text { Continuous Monitor- } \\
\text { ing (ISCM) }\end{array}$ & $\begin{array}{l}\text { Maintaining ongoing awareness of information security, vulnerabilities and } \\
\text { threats to support organizational risk management decisions. }\end{array}$ & $\begin{array}{ll}\text { SP } & 800-137 \\
{[15]} & \end{array}$ \\
\hline Risk & $\begin{array}{l}\text { Effect of uncertainty on objectives. A positive or negative deviation from } \\
\text { what is expected. }\end{array}$ & ISO 31000 [16] \\
\hline Risk Assessment & $\begin{array}{l}\text { The process of identifying, prioritizing and estimating risks. This includes } \\
\text { determining the extent to which adverse circumstances or events could im- } \\
\text { pact an enterprise. Uses the results of threat and vulnerability assessments to } \\
\text { identify risk to organizational operations and evaluates those risks in terms } \\
\text { of likelihood of occurrence and impacts if they occur. }\end{array}$ & CNSSI-4009 \\
\hline Security Label & $\begin{array}{l}\text { Information that represents or designates the value of one or more security } \\
\text { relevant-attributes (e.g., classification) of a system resource. }\end{array}$ & CNSSI-4009 \\
\hline Security Metrics & $\begin{array}{l}\text { Tools designed to facilitate decision making and improve performance } \\
\text { and accountability through collection, analysis and reporting of relevant } \\
\text { performance-related data. IT security metrics must be based on IT security } \\
\text { performance goals and objectives. }\end{array}$ & $\begin{array}{l}\text { NIST SP } 800- \\
55[17]\end{array}$ \\
\hline Security Testing & $\begin{array}{l}\text { Process to determine that an information system protects data and maintains } \\
\text { functionality as intended }\end{array}$ & CNSSI-4009 \\
\hline Threat & $\begin{array}{l}\text { Any circumstance or event with the potential to adversely impact organiza- } \\
\text { tional operations, organizational assets or individuals through an information } \\
\text { system via unauthorized access, destruction, disclosure, modification of in- } \\
\text { formation and/or denial of service. }\end{array}$ & FIPS 200 \\
\hline $\begin{array}{l}\text { Target of Evaluation } \\
\text { (TOE) }\end{array}$ & $\begin{array}{l}\text { An information technology product or system and associated administrator } \\
\text { and user guidance documentation that is the subject of an evaluation. }\end{array}$ & FIPS 140-2 [18 \\
\hline Vulnerability & A weakness that can be exploited by one or more threats & ISO 27000 [19] \\
\hline
\end{tabular}

proposed in the ARMOUR project. In this sense, it should be noted that the proposed approach is part of the methodology that was implemented and developed in the Horizon 2020 ARMOUR project3. which was mainly focused on automating the security testing activities. The automation of this process is crucial to support the dynamism inherent to security, specially in IoT scenarios, and to maintain updated the security level of IoT devices. One device can be secure today, but tomorrow, a new vulnerability can cause it to be insecure.

The remainder of this paper is structured as follows. In Section 2, we study the main challenges associated to the creation of this certification framework, and the current efforts towards this end. In Section 3 we explain the mentioned ETSI approach and the proposed instantiation based on specific tools. The application of the resulting methodology to a concrete IoT scenario is explained ${ }_{60}$ in Section 4 . In Section 5 we analyse our approach, proposing some future lines of work. Finally, Section 6 summarizes the conclusions obtained.

For the sake of the understandability, Table 1 provides the definition of the main terms used in this paper.

\footnotetext{
${ }^{3}$ http://www.armour-project.eu
} 


\section{Current efforts towards an IoT security certification framework}

65 systems, domains, solutions, services, organizations or people (e.g., training and certification) derives on a heterogeneous landscape of solutions. Consequently, for different stakeholders it is difficult to understand the requirements to achieve a certain level of security in each context or technology. This heterogeneity also makes the comparison of certified devices more difficult, countries and contexts. Currently, there is not a unified solution that copes with these issues; therefore, the process of comparing and assessing the security levels of different IoT deployments is challenging. This section analyses some of the main certification schemes. Table ?? presents the advantages and disadvantages of each one of them. It should be pointed that there is only one

\begin{tabular}{lll}
\hline $\begin{array}{l}\text { Certification } \\
\text { Scheme }\end{array}$ & Advantages & Disadvantages \\
\hline CC & $\begin{array}{l}\text { It represents the main standard for certifi- } \\
\text { cation nowadays. Furthermore, it allows } \\
\text { products to be updated during the life- } \\
\text { time of certification considering different } \\
\text { layers and components to be certified. }\end{array}$ \\
\hline \hline CPA & Madrid & Ciudad \\
\hline CAP UL & Sevilla & Ciudad \\
\hline CSPN & Pars & Ciudad \\
\hline ULD & Pars & Ciudad \\
\hline ICSA & Pars & Ciudad \\
\hline
\end{tabular}

Table 2: Tabla muy sencilla.

The current main security certification standard is the Common Criteria (CC) [20], in which the security functional and assurance requirements are specified through Protection Profiles (PPs) for a Target of Evaluation (TOE), which is a set of software, firmware and/or hardware components. However, it does not include security risk assessment on evaluation results, so the result is binary (i.e., it fulfils the profile or not). It uses Evaluation Assurance Levels (EAL) to describe numerically the depth and rigor of an evaluation. CC describes the set of general actions the evaluator has to carry out, but it does not specify procedures to be followed for those actions. CC provides assurance that the process of specification, implementation and evaluation of a product has been conducted in a rigorous, standard, and repeatable manner. Even if CC is the main standard and it is well developed, a number of limitations has been identified [21] [22], which are being taken into account by the CC community, such as the time and effort required to execute an evaluation especially for a high EAL, or the management of changes in the certified product. If the product is still in the growing phase from the market point of view, this cost can become a serious obstacle for commercialization (especially in IoT). CC has also a problem of lack of comparability, due to the 9 difficulty in understanding the CC technical documents for the certification of a product, which makes an objective comparison more difficult [23]. In addition, CC certificates a particular version of the product in certain configurations. Any changes to the configuration or any updates to the 
product that affect the TOE may invalidate the certification. This is not a desirable situation, given that products evolve and are updated at a frantic pace and the certification must not be frozen to a specific version of the product. However, despite some disadvantages, CC is the main security certification standard, widely recognized and developed, so for the homogeneity of the terms, our proposal reuses the concepts of EAL and TOE.

Other important schemes are the Commercial Product Assurance (CPA) [24], the Cybersecurity Assurance Program (UL CAP) [25], the Certification de Scurit de Premier Niveau (CSPN) [26] or the ULD Datenschutz-Gtesiegel [27]. In first place, CPA represents an approach from the Communications-Electronics Security Group (CESG) in the UK, to increase the level of trust regarding security aspects of commercial products. However, while it was intended to replace other approaches such as CC, there is no Mutual Recognition Agreement (MRA) for CPA. This basically means that products tested in the UK will not normally be accepted in other markets. cybersecurity criteria to assess vulnerabilities and weaknesses in connected devices. However, due to its recent creation, it is not widely recognized as an accepted certification scheme yet. The CSPN was created by the National Cybersecurity Agency of France (ANSSI) in 2008. A significant point to be highlighted is that CSPN aims at performing evaluations in a short period through the adaptation of the product development life cycle, addressing one of the challenges mentioned before. However, some of the most important issues such as the dynamism or the labelling are not mentioned. Finally, the scope of the ULD Datenschutz-Gtesiegel encompasses IT products in general, i.e., hardware, software, automated processes and services. A prerequisite is that they are suitable for use by public authorities. It certifies that the compatibility of a product with the thes on data protection and data security has been established in a formal procedure. Although for minor changes, a simplified certification is only required, for fundamental changes, the overall process must be repeated.

Although there is a large variety of general certification approaches, specifically for IoT the proposals are very limited, and they do not address all the challenges directly associated with these type of devices and with the management of the security during their lifecycle. The only one that considers certification is the ICSA Labs IoT Security Testing Framework[28], focused on specifying security testing requirements for distinct classes of IoT device types. Although it includes frequent iterative updates and security monitoring during the life cycle, it is not addressed the automation of the process (it uses a manual checklist) and the labelling is not considered. Other security frameworks not considering certification are the IoT Security Compliance Framework[29] and the IoT Trust Framework[30]. The first one provides a questionnaire based upon the best practices, allowing to test for compliance, and taking into account specific IoT characteristics such as the transfer of ownership for IoT devices and sensitive data lifecycle management, whereas the latter includes a set of strategic principles to help secure IoT devices throughout their entire life-cycle.

A security certification framework should address the different stages of an IoT devices life cycle [31]. On one hand, the security of the device should be monitored during the whole life cycle, in order to identify new potential vulnerabilities. On the other hand, the cybersecurity certificate (and the associated label) should be updated, when a cybersecurity recertification process is required because of a security change, understood in terms of a new vulnerability or threat applicable that causes the product to be less secure than before, or because of an update/patching of the product. Indeed, security is itself a very dynamic concept; at the end of the certification process, a device could be secure, but this condition can change at any time [31, 32, 33]. This makes a lightweight recertification process very relevant for a successful approach. However, the existing proposals are 
usually expensive, slow and complex, requiring formal documentation and processes [33] [32].

Another key point is that a system composed by IoT devices could be composed by several components with different levels of security, so the aggregation of these different security levels could be required. Another related challenge is the aggregation of the security related to each layer from the IoT protocol stack, by considering different vulnerabilities at each layer. This aggregation should be done in an objective way, by considering the use of consistent and well-known security metrics. However, some of the metrics used in the current schemes, such as likelihood or impact, are difficult to be measured due to its complexity, which is reported in [23]. Finally, the context in which the device will operate must be considered to specify the boundary conditions where the security certification was applied, and the resulting cybersecurity label should provide a clear visibility of the security achieved, as recommended in [34].

While some of the previous challenges are being currently addressed by European organizations, the dynamic context where IoT devices are deployed and operate makes the adoption of a cybersecurity certification framework challenging. In this sense, DIGITALEUROPE has published some recommendations for Cybersecurity Certification and Labelling Scheme, such as a dynamic cybersecurity label, self-certification, global support, test automation and cost effective process [6]. ECSO [5] has also done a wide state of the art focusing on standards that can be (potentially) used as the basis for assessing the overall cybersecurity of a product or component, an ICT service, a service provider, organization or a critical infrastructure.

Indeed, based on some of the main recommendations pointed out by ECSO [35], security assessment and testing are considered as crucial aspects to foster the creation of a certification framework. In this sense, the proposed IoT specific cybersecurity certification approach (in the scope of the Horizon 2020 ARMOUR project) is intended to provide a more standardized view based on the ETSI security risk assessment and testing methodology. In particular, our proposal is built on top of both processes as a way to provide feedback between them and helping to refine the results provided from each process. Furthermore, the definition of our proposal is also influenced by ECSO guidelines; specifically, our proposed security label is intended to provide a multidimensional and visual representation of a device's security level. This label could be considered to be part of the security certificate (e.g. the European Cyber Security Certificates (ECSC) from ECSO) embracing other aspects of the certification process that are considered in the context of the recent Cybersecurity Act in Europe [36.

In the next subsections, we analyse the different existent approaches for the two main processes considered as part of the certification proposal: security risk assessment and testing.

\subsection{Security Risk Assessment}

As part of the certification process, being able to measure the risk of different IoT security approaches or solutions is crucial to quantify their security level. It provides a numeric value for the risk that allows to compare different configurations and scenarios in an objective and easy way. There is a high number of security risk assessment methods managed by both commercial and non-commercial organizations. However, they are often subjective (such as the SANS 4 vulnerability analysis scale or the DREAD scheme [37]), specific for web applications (e.g., the OWASP Application Security Verification Standard (ASVS) Projec (5), or too large and complex [38], such as OCTAVE [39].

\footnotetext{
${ }^{4}$ https://www.sans.org

${ }^{5}$ https://www.owasp.org
} 
Moreover, the Common Vulnerability Scoring System (CVSS) [9] represents a widely established approach; for example, it is used together with the Common Vulnerabilities and Exposures (CVE) CVSS follows a similar process to the Common Weakness Scoring System (CWSS) [40] by considering three groups of metrics; in particular, the Base metrics produce a score from 0.0 to 1.0, modified by the optional Temporal and Environmental metrics. CVSS has been widely adopted, especially the use of base scores from the Base metric group. Each metric in this group is assigned a value, and these values are converted to associated weights, and applied to a formula in order to calculate the base subscore. These approaches are well defined and their metrics mostly comprise the metrics used by other security risk assessment methods previously described.

Conceptually, CVSS and CWSS are quite similar; indeed, we recommended the usage of CWSS in our previous work [12]. However, although CWSS provides a more extended set of metrics, and the possibility of establishing an unknown or default value for a metric, its complexity makes the mapping between the testing and assessment processes more difficult. We have chosen CVSS based on its simplicity since CWSS has more metrics than CVSS and some of them, like the likelihood, are difficult to measure, as reviewed before. In addition, the certification is intended to be applicable over a vulnerability database, and, although both of them are used for this purposes, CVSS is being used in the National Vulnerability Database (NVD) 7 created by the NIST. However, it should be pointed out that a specific instantiation of the ETSI proposal is considered in this work; indeed, a different combination of approaches and models could be also valid.

\subsection{Security Testing}

Being able to test the security of the different IoT security approaches allows to prove the security level assigned to them. Some of the current security testing approaches are briefly described below [41].

Penetration testing [42] [43] is similar to an actual attack from a malicious third party, with limited information about the system under test and only able to interact with the system's public interfaces. This technique is generally manual and it is combined with the usage of black-box vulnerability scanners, which are used to identify security issues in applications.

Fuzzing testing [44] is a technique consisting on passing into a target system valid and invalid message sequences to see if the system breaks, and if it does, what makes it break. An important system. This type of technique is very useful to test injection attacks for example, and can be combined with other testing mechanisms.

Moreover, regression testing [45] techniques are focused on the update process of the device, ensuring that changes do not cause unintended effects on unchanged parts and changed parts of the software behave as intended.

Usage-based testing [46] focuses the usage of a system. Instead of testing all parts of the system equally, the parts that are often used are tested intensively, while seldom or never used parts are ignored. There is also a combined version with fuzzing proposed in [47].

The Risk-based security testing [43] approach tries to improve security testing with the help of security risk analysis and the final results are test result reports. There are different methods, some of them trying to identify test cases whereas others try to prioritize test cases.

\footnotetext{
${ }^{6}$ https://cve.mitre.org/

${ }^{7}$ https://nvd.nist.gov/
} 
Finally, code-based testing [41] detects vulnerabilities by looking at the code. This can be performed manually or automatically using a specific tool.

However, compared to traditional testing methods, MBT is able to manage and accomplish testing tasks in a more cost effective and efficient way. A model represents the system under test, its environment, or the test itself, which directly supports test analysis, planning, control, implementation, execution and reporting activities. In addition, a large number of MBT tools have been developed to support the practice and utilization of MBT technologies in real cases [48]. The use of MBT and tools such as TITAN or Certify in the scope of the EU ARMOUR project allows automating the security testing, coping with one of the main challenges associated to security, the dynamism, in the sense that if a security change occurs (new discovered vulnerability, new security configuration, patch, update, etc.), it can be quickly reflected in the label.

It should noted that the testing methods can be also combined to create new testing methods (e.g., MBT with fuzzing [49]) or complementing each other in different phases of the device's life cycle [41].

\subsection{The ETSI Risk-based Security Assessment and Testing Methodologies}

The ETSI proposal [7] combines an extended security assessment derived from ISO 31000 and typical security testing activities following the standard ISO 29119. This methodology was initially developed and evaluated in the RASEN [50] research project.

We consider the definition of a cybersecurity certification framework to be built on top of two main streams of this proposal: Security Testing, which is intended to discover flaws, vulnerabilities or other technical issues, and Security Risk Assessment, which is meant to analyze potential vulnerabilities addressing legal or business issues. In addition, an initial process Establishing the Context is included to set up both processes and to analyse the context where the device or component is being tested. This process is associated with understanding the business, the regulatory environment, the laws and analysing which security level is required in each of them. It also includes the test planning, which is focused on developing the test plan (objective, scope, order, testing technique etc.). The activities Monitoring $\mathcal{E}$ Review, and Communicate $\mathcal{E}$ Consult are intended to set up the management perspective, continuously reacting and controlling the information derived from assessment and testing processes. Finally, the Treatment phase is intended to provide security countermeasures taking into account the risk observed.

The proposal distinguishes two main perspectives, a test-based risk security assessment (Figure 1. a) and a risk-based security testing (Figure 1, b). In the test-based risk security assessment, testing is used to guide and improve the security risk assessment, adjusting risk values and providing

5 feedback. In case of the risk based security testing, security risk assessment results are used to drive the testing, and to prioritize the areas to be tested according to their risk.

Following the ETSI proposal, Security Risk Assessment is composed by three main activities:

- Risk Identification. It comprises a set of activities of finding, recognizing and describing risks, as well as identifying their causes and potential consequences.

- Risk Estimation. It determines the level of risk, understanding its sources and its consequences.

- Risk Evaluation. It compares the results of risk estimation with risk criteria to determine whether the risk and/or its magnitude is acceptable or tolerable.

In the same way, Security Testing is also composed by three activities: 

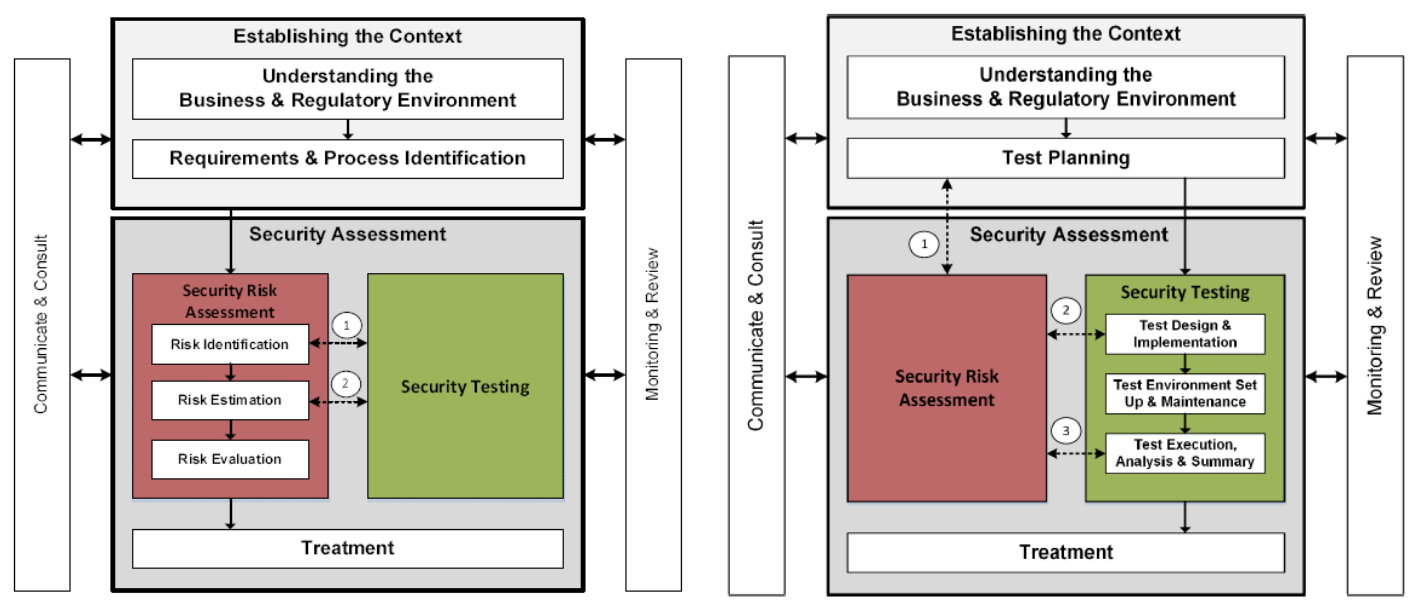

Figure 1: a) ETSI test-based risk security assessment proposal [7]. (b) ETSI risk-based security testing proposal [7].

- Test Design and Implementation. It generates the test cases, implemented and assembled to test procedures.

- Test Environment Set Up \& Maintenance. This involves establishing and maintaining the environment in which tests are executed.

- Test Execution, Analysis \& Summary. It deals with the test execution as well as with the systematic analysis and summary of test results.

Following the steps considered within the RASEN project [51], this work proposes the use of specific technologies and tools for security risk assessment and testing processes, as the main building blocks to build a security certification and labelling approach for IoT devices. As described in Section 3 it represents, in turn, an instantiation of the described methodology proposed by ETSI, as a way to avoid reinventing the wheel and to take advantage of its standardized basis.

This approach is intended to fill the gaps of an IoT certification scheme, discussed before. In this sense, the labelling must be considered an important step in the process. This label must be be considered dynamic to be in line with the current security level of the device, and the recertification process must be the most automated possible in order to update the label in an easy, fast and non expensive way, coping with the time, complexity, cost and scalability challenges. In this sense, test generation and execution can be automated, as well as the risk estimation. Nevertheless, to be able to automate the risk estimation, the metrics must be objective enough to avoid the presence of a human during the estimation. The context in which the device will be operating should be taken into account in some way during the process, either in the risk estimation or in the labelling. This will also help to compare different devices objectivity depending on the context, since different contexts require different security levels. All the process should be based on standards to benefit from the long analysis that has taken place on the part of the community and to promote its acceptance.

Finally, the process must review the security during the whole life cycle of the IoT device. This can be achieved through the recertification and the dynamic label, that allows the management 
of changes on the security level during its life. Nevertheless, to be able to detect these changes, a monitoring process must be considered.

\section{Proposed approach for a Cybersecurity Certification Framework}

As mentioned before, the proposed IoT specific cybersecurity certification approach is based on the ETSI security risk assessment and testing methodology. We consider the automated testing approach from the ARMOUR project, in order to face the IoT challenges related with dynamism and scalability. While the definition of this cybersecurity certification framework needs to address significant challenges (as previously described), the proposed approach is intended to serve as the baseline for a more harmonized and consistent view of the certification process for IoT.

Figure 2 shows the overall process of certification that is derived from ETSI proposal based on ISO 31000 and ISO 29119, and extended to include specific activities related to certification. In particular, a labelling activity has been integrated within the Communicate $\mathcal{E}$ Consult process, so that it is in charge of communicating the security level obtained for a specific Target of Evaluation (TOE). In addition, although the original methodology includes a Treatment process (i.e., security controls and other countermeasures), this is not addressed in our instantiation. However, treatment activities can be designed from the results of the security testing and risk assessment processes. 


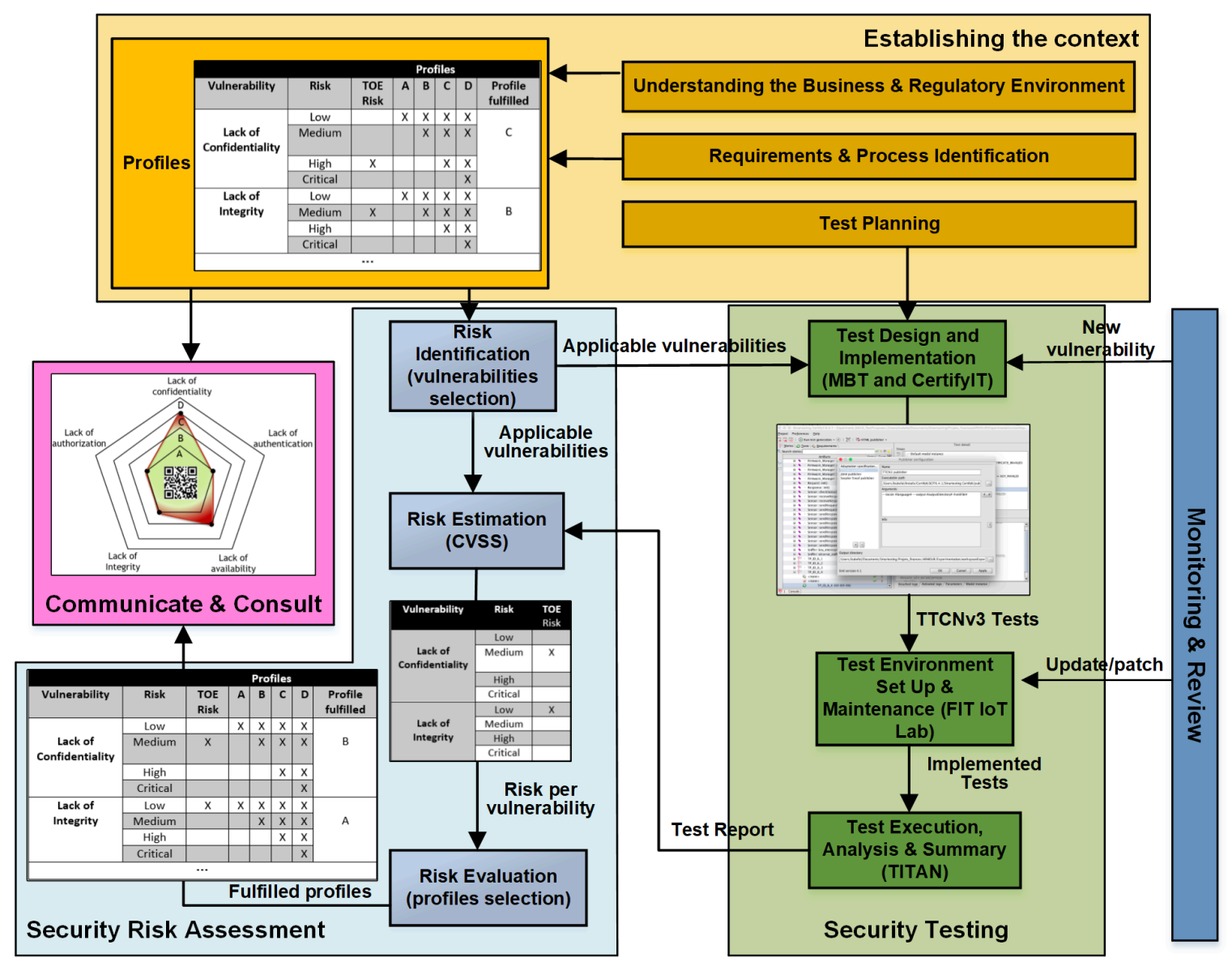

Figure 2: General overview of the certification process based on the ETSI proposal

As already mentioned, the proposed certification approach is intended to certify a certain TOE. According to CC, a TOE is defined as a set of soft-ware, firmware and/or hardware possibly accompanied by guidance. In our approach, we consider that the TOE also includes a specific configuration (i.e., a concrete protocol, library used, security parameters, etc.) and the context in which it is intended to operate.

Before describing the main process, it should be noted that the certification approach takes a set of vulnerabilities applicable to a certain TOE as a starting point. In particular, the NVD is used to find vulnerabilities previously discovered in the TOE. Furthermore, the set of generic oneM2M [52] vulnerabilities is used for testing purposes, in order to discover zero-day vulnerabillities for the TOE. Then, the resulting set of vulnerabilities is mapped to five general security properties; Confidentiality, Integrity, Availability, Authentication and Authorization:

- $\quad$ Lack of authentication. The endpoints should be legitimate.

- $\quad$ Lack of integrity. Received data are not tampered with during transmission; if this does not happen, then any change can be detected. 
- $\quad$ Lack of confidentiality. Transmitted data can be read only by the communication endpoints.

- $\quad$ Lack of authorization. Endpoint services should be accessible to endpoints who have the right to access them.

- Lack of availability. Exceptions should be controlled to avoid faults that affect the endpoints.

The mapping has been done following the Table 3 , where the relation between them is specified in the second column. The vulnerability 12 (context awareness) is intended to be reflected in the profiles defined in the next section. It should be noted that the proposed mapping provides a more simplified view of the security aspects based on our previous work [12]. Indeed, the five properties have been extracted from some of the most referenced security aspects that can be found in current IoT literature [53] [54] [55]. In this way, the proposed approach is designed to measure the risk associated with the lack of each property, so the resulting security label can be specified by using such properties to provide a multidimensional security description.

Table 3: Relation between OneM2M vulnerabilities and the five general vulnerabilities considered in the proposal

\begin{tabular}{|c|c|c|}
\hline General vulnerabilities & Relation & TOE-independent vulnerabilities (0neM2M) \\
\hline \multirow{5}{*}{ Lack of authentication } & Protection against a device with non valid ID & V10,V14 \\
\hline & Protection against a device with a valid ID but non valid authentication key or certificate & V3,V13 \\
\hline & Cryptographic suite & $\mathrm{V} 1, \mathrm{~V} 4, \mathrm{~V} 19$ \\
\hline & Protection against a server with non valid ID & $\mathrm{V} 10, \mathrm{~V} 6, \mathrm{~V} 14$ \\
\hline & Protection against a server with a valid ID but non valid authentication key or certificate & $\mathrm{V} 4, \mathrm{~V} 13$ \\
\hline \multirow{3}{*}{ Lack of confidentiality } & Percentage encrypted (general) & V7,V13 \\
\hline & Cryptographic suite & V19 \\
\hline & Percentage encrypted (related with keys) & V6 \\
\hline \multirow{3}{*}{ Lack of authorization } & Different profiles per device & V18,V10 \\
\hline & Cryptographic suite and key length & V19 \\
\hline & Protection against a replacement with a more privileges key & V3 \\
\hline \multirow{2}{*}{ Lack of integrity } & Percentage of integrity protection & $\mathrm{V} 8, \mathrm{~V} 13, \mathrm{~V} 9$ \\
\hline & Cryptographic suite & V19,V17 \\
\hline \multirow{6}{*}{ Lack of availability } & Low cascade impact & V11 \\
\hline & Exception control against buffer overflow & V15 \\
\hline & Protection against injection attacks & V16 \\
\hline & Control the input data & V20 \\
\hline & Control scripts & V21 \\
\hline & DoS attack & $\mathrm{V} 2, \mathrm{~V} 14, \mathrm{~V} 5, \mathrm{~V} 3$ \\
\hline
\end{tabular}

It should be pointed out that we adapt the ETSI concepts and processes introduced in Section 2.3 to our proposed cybersecurity framework. In this sense, the first process, which is called establishing the context is related with understanding the business and regulatory environment, and analyse which security level is required in each of them (requirements and process identification). For example, in a medical context, confidentiality and availability could be considered two very important security properties that could not be so important in a domestic context. As a result of this process, several security profiles (e.g., A, B, C, D) related to the context will be defined. The last activity of the first process, the test planning, is the activity of developing the test plan (objective, scope, order, testing technique etc.). In this activity, the techniques of testing are chosen regarding each vulnerability, as well as the order of the tests and their scope.

The security assessment process includes the security risk assessment and the security testing. Within the security risk assessment, we have three activities. The risk identification uses vulnerability databases (e.g., the NVD) and oneM2M vulnerabilities as input. Taking into account the specific TOE, this activity is in charge of selecting which vulnerabilities will be tested. It should be pointed that although no vulnerability from the vulnerability databases was applicable, the basic tests defined from the oneM2M vulnerabilities in Table 3 make possible to label the device with objective and 
test gathered data. The Risk estimation assigns a risk mark to each vulnerability. For this purpose, process are provided. Finally, risk evaluation compares the result of the risk estimation with the profiles considered in the establishing the context process. In this way, the TOE obtains a profile for each security property.

The security testing process is related to the creation and execution of security tests. However, in the RASEN project proposal [51], the automation of this process is not fully contemplated. In this sense, the proposed instantiation is intended to use specific technologies to help for automating this process, easing the update of the cybersecurity label to cope with changing conditions in which the device operates. The integration of such approaches has been done in the scope of the ARMOUR project. The first activity included within this process, test design and implementation, set up and maintenance, the environment where the tests will be executed is set up. Then, in test execution, analysis and summary, the implemented tests are executed. From this execution, we gather information related with the results of the tests that are used for risk estimation.

Furthermore, Figure 2 shows additional support activities like communicate and consult and the device security. The latter is meant to gather information from inside (risk, context, etc.) and outside (experts, databases, laws, etc.) the process, and to communicate it in an appropriated way. As already mentioned, this activity has been enriched with the labelling process. This way, with the data collected from the test execution, taking into account the profile obtained and the context, the certification process generates a cybersecurity label, helping the user to know the security level of the TOE. This label is intended to be part of the whole security certificate associated to a specific TOE.

The following sections are intended to describe the instantiation of the ETSI architecture by using concrete approaches and technologies. Although this paper is focused on the two main processes, security testing and security risk assessment, we also provide a general overview of the other processes, which we further considered in our future work in this area.

\subsection{Establishing the context}

This first process is composed by three activities that aim to set up the certification process. In the first activity, understanding the business and regulatory environment, a risk analysis is performed in order to determinate which level of security is needed in a specific domain. Experts in each context could be required to perform this analysis. It includes understanding the laws and the regulation environment. The set of regulations (including GDPR for data) and stakeholders requirements would be processed to create sets (for different levels) of baseline requirements. The requirements are linked to the types of data to be produced. For example, location data will have specific requirements in the overall set of requirements (which will be defined in the related protection profile for that domain). Then, the domain and the data sets will be linked, adding the context variable to the cybersecurity label, which will be reflected in the profiles associated to each of them, coping with one of the challenges previously described.

From this analysis, the next activity, requirements and process identification, defines several profiles (A, B, C, D), (e.g., in a similar way to the European energy label [56]). The number of profiles can be modified to make the security level more accurate. The profiles indicate which level of security must be achieved by the TOE in each vulnerability considered and for a specific context to obtain each profile, following the notation of Table 4 . In this example, a TOE obtains the A profile if it has 
a low risk level in confidentiality. It is worth noting that if a TOE fulfils a specific profile, it also fulfils the lower ones (e.g., if A profile is obtained, it also fulfils B, C and D profiles).

Once the requirements of each domain are set up, the next step is planning and defining the tests. Test planning phase includes analysing the security of the device and design what tests should be implemented. Although it is not considered in this activity, planning could be used to prioritize the tests in order to perform a fast regression testing in case of a recertification process.

Table 4: Example of profile definition

\begin{tabular}{cccccc}
\hline Vulnerability & Risk & A & B & C & D \\
\hline \multirow{4}{*}{ Lack of confidentiality } & Low & x & x & x & x \\
& Medium & & x & x & x \\
& High & & & x & x \\
& Critical & & & & x \\
\hline \multirow{4}{*}{ Lack of integrity } & Low & x & x & x & x \\
& Medium & & x & x & x \\
& High & & & x & x \\
& Critical & & & & x \\
\hline & ... & & & & \\
\hline
\end{tabular}

\subsection{Security testing}

From the vulnerabilities considered to be applicable to the TOE, different security tests are produced. These tests are used to find out new vulnerabilities in the TOE, as well as to obtain different results to generate a test report with data associated with the security level.

This way, during the test design and implementation, a test suite is designed to test the risk's grade of each vulnerability. To automatize this process, a MBT approach is used to specify the tests and their behavior. MBT has shown its benefits and usefulness for systematic compliance testing of systems [57]. In this approach, the structure of the system is modelled by Unified Modelling Language (UML) class diagrams, while the system behavior is expressed in Object Constraint Language (OCL) 8 , using the CertifyIt tool. Functional tests are obtained by applying a structural coverage of the OCL code describing the operations of the IoT system including devices and protocols (TOE). We export the tests defined in MBT to Testing and Test Control Notation (TTCN) v.3 language using the tool CertifyIt. The main goal of the use of TTCN-3 in the proposed approach is the systematic and automatic testing of security properties in IoT devices for improving efficiency and scalability. To cope with the particularities of each IoT device, we use adapters, a middle interface between the TTCN-3 and the device code.

Secondly, in the test environment set up and maintenance phase, we set up the environment in which the tests will be executed. For this purpose, the proposed instantiation considers the use of FIT IoT-LAB. This platform consists of a large-scale infrastructure (with about 2000 IoT nodes) for testing purposes without the need of cumbersome deployment tasks.

Finally, in the test execution, analysis and summary phase, we execute the tests on a local or external large-scale testbed (e.g., FIT IoT Lab), where we test the implemented scenario by means of TITAN. TITAN is a TTCN-3 compilation and execution environment for different platforms that

\footnotetext{
${ }^{8}$ http://www.omg.org/spec/OCL/2.4
} 


\begin{tabular}{ll}
\hline Metric & Summary \\
\hline Attack vector (AV) & $\begin{array}{l}\text { This metric reflects the context by which vulnerability exploitation is possible. This metric value (and consequently the Base score) will be larger the more remote } \\
\text { (logically, and physically) an attacker can be in order to exploit the vulnerable component. }\end{array}$ \\
\hline $\begin{array}{l}\text { Attack Complexity } \\
\text { (AC) }\end{array}$ & \begin{tabular}{l} 
This metric describes the conditions beyond the attackers control that must exist in order to exploit the vulnerability. \\
\hline $\begin{array}{l}\text { Privileges Required } \\
\text { (PR) }\end{array}$
\end{tabular} \\
$\begin{array}{ll}\text { User } \quad \text { This metric describes the level of privileges an attacker must possess before successfully exploiting the vulnerability. } \\
\text { (UI) }\end{array}$ & $\begin{array}{l}\text { This metric captures the requirement for a user, other than the attacker, to participate in the successful compromise of the vulnerable component. It determines } \\
\text { whether the vulnerability can be exploited solely at the will of the attacker, or whether a separate user must participate. }\end{array}$ \\
\hline Scope (S) & $\begin{array}{l}\text { Scope refers to the collection of privileges defined by a computing authority when granting access to computing resources. These privileges are assigned based on } \\
\text { some method of identification and authorization. }\end{array}$ \\
\hline $\begin{array}{l}\text { Confidentiality Im- } \\
\text { pact (C) }\end{array}$ & $\begin{array}{l}\text { This metric measures the impact to the confidentiality due to a successfully exploited vulnerability. Confidentiality refers to limiting information access and disclosure } \\
\text { to only authorized users, as well as preventing access by, or disclosure to, unauthorized ones. }\end{array}$ \\
\hline Integrity Impact (I) & \begin{tabular}{l} 
This metric measures the impact to integrity of a successfully exploited vulnerability. Integrity refers to the trustworthiness and veracity of information. \\
\hline $\begin{array}{l}\text { Availability Impact } \\
\text { (A) }\end{array}$
\end{tabular} $\begin{array}{l}\text { This metric measures the impact to the availability of the impacted component resulting from a successfully exploited vulnerability. This metric refers to the loss of } \\
\text { availability of the impacted component itself, such as a networked service. }\end{array}$ \\
\hline
\end{tabular}

in combination with CertifyIt create executable tests, whereas FIT IoT-LAB offers the large-scale testbed on which the test cases are executed. TITAN sends the test commands (TTCN-3) to the device through the adapter implemented before in order to execute the tests.

The results of the tests (i.e., the test report) will help to establish the security level (through the security label) in a more refined way, since they are used during the security risk assessment process, allowing to measure the risk associated to each vulnerability.

With the automation of this process, if a new vulnerability is discovered, the recertification process can be done in a cost and time effective way, which is key to address the dynamic nature of cybersecurity in IoT. It is worth noting that the proposed mechanisms for the automation have been considered within the ARMOUR project, but they are independent of the methodology used to automate the process.

\subsection{Security risk assessment}

Security risk assessment process is intended to provide a refined risk mark for each vulnerability taking into account the test report obtained from the testing process. The risk mark obtained is used through the certification process to obtain the cybersecurity label as part of the certificate to be generated. In particular, this mark is used to select the profile fulfilled by the TOE from the set of profiles that are established during the establishing the context process.

The security risk assessment is composed by three main activities. In the risk identification, from the vulnerabilities considered in the establishment of context process, the set of vulnerabilities applicable to the TOE are identified. In order to be able to obtain the profile that is used for the labelling, in the risk estimation we obtain a risk mark associated to each vulnerability considered. According to Section 2. CVSS has been chosen to perform the security risk assessment with its specific metrics, as shown on Table5[9].

Furthermore, we calculate the base score for each vulnerability by means of the CVSS formula. In case the Impact subscore $\left(I_{S}\right)$ is negative, the base score will be zero. If scope is unchanged, then

$$
\begin{gathered}
\text { Base }=\left[\min \left\{I_{S}+E_{S}, 10\right\}\right] \\
I_{S}=6.42 \cdot I S C
\end{gathered}
$$

else

$$
\text { Base }=\left[1.08 \cdot \min \left\{I_{s}+E_{s}, 10\right\}\right]
$$


Table 6: CVSS risk intervals [9]

\begin{tabular}{ll}
\hline Rating & CVSS Interval \\
\hline None/Low & $0-3.9$ \\
Medium & $4.0-6.9$ \\
High & $7.0-8.9$ \\
Critical & $9.0-10.0$ \\
\hline
\end{tabular}

Table 7: Evaluation of a TOE

\begin{tabular}{|c|c|c|c|c|c|c|c|}
\hline & & CVSS & Prc & ile & & & Profiles fulfilled \\
\hline Vulnerability & Risk & TOE & A & B & C & $\mathrm{D}$ & TOE \\
\hline \multirow{4}{*}{ Lack of integrity } & Low & \multirow{4}{*}{$x$} & \multirow[t]{4}{*}{$\mathrm{x}$} & $\mathrm{x}$ & $\mathrm{x}$ & $\mathrm{x}$ & \multirow{4}{*}{ B } \\
\hline & Medium & & & \multirow[t]{3}{*}{$\mathrm{x}$} & $\mathrm{x}$ & $\mathrm{x}$ & \\
\hline & High & & & & $\mathrm{x}$ & $x$ & \\
\hline & Critical & & & & & $\mathrm{x}$ & \\
\hline
\end{tabular}

$$
I_{S}=7.52 \cdot(I S C-0.029)-3.25(I S C-0.02)^{15}
$$

where

$$
\begin{gathered}
E_{S}=8.22 \cdot A V \cdot A C \cdot P R \cdot U I \\
I S C=1-((1-C) \cdot(1-I) \cdot(1-A))
\end{gathered}
$$

Finally, in the risk evaluation activity, to obtain the profile fulfilled, we use the mapping defined in CVSS between the numeric risk value and each one of the four risk levels (none-low, medium, high and critical), as shown in Table 6

We determine the profile comparing the results obtained in the security risk assessment with the profiles available for the specific context, choosing always the highest profile fulfilled for each vulnerability. For example, in Table[7]a TOE has obtained a Medium risk level in lack of integrity, so it obtains B, C and D profiles. However, it will obtain the highest one, in this case the B profile. This process is repeated for all the vulnerabilities.

As the profile is an important part of the cybersecurity label, this process has a close communication with the labelling activity, providing the results from the assessment.

\subsection{Monitoring and review}

The main concept of a certification monitoring system for cybersecurity is to design, develop and deploy a system, which is able to collect and correlate events and data from the devices and systems to detect security changes, due to new security vulnerabilities, intrusion attacks, the exploitation of security vulnerabilities, an updating, a patch a change on the context of the device, etc.

Although the instantiation of this activity is not addressed in our proposal, it is used to detect both expected security changes (updates and patches) and unexpected security changes (new discovered vulnerabilities). In this sense, there should be a monitoring activity of the database containing the IoT vulnerabilities to detect a new one and to assess if the vulnerability is applicable 
to the device being monitored. In addition, there should be also a monitoring activity of the patches and updates performed by the manufacturer and a monitoring of the changes produced by the user, that is, re-ownering (change of owner), changes on the scalability, etc.

If the monitoring and review detects a new vulnerability, the test design activity is in charge of model it, incorporating this new event in the security risk assessment and producing a new label. If the detected event is already contemplated, the monitoring and review starts the test execution activity in order to verify if there has been a change in the test report, leading or not to a new security level. Another scenario could be that the event causes a new applicable vulnerability not considered before; in this situation, the tests report is used to discover it and be used as input for the risk identification activity.

Monitoring and review can also have a vulnerability discovering function by means of the usage of scanning tools. In this case, when the scanning process inside the monitoring and review process detected a new vulnerability, the recertification process is performed in order to update the resulting label. In case a new vulnerability is discovered, the general database could be updated, and the resulting value from security risk assessment could be used to give an approximated risk value for the vulnerability.

\subsection{Security Labelling}

One of the main results of the certification process is a security label associated to the risk being obtained for a specific TOE. As already mentioned, the proposed label considers the TOE definition and the profiles fulfilled for each general vulnerability. It should be noted that, according to ECSO recommendations [35], the resulting label is intended to be part of the security certificate. This certificate the EALs from CC to indicate how the certification process itself has been performed (self-certification, third party, etc.).

For security labelling, there is a tradeoff between the simplicity required for the understanding by a non-expert consumer, and the information presented in the cybersecurity label. Following the recommendations of ENISA [4], as security requirements are in fact multi-dimensional, the cybersecurity label includes the profile of each general vulnerability considered. In this way, the user is provided with more information and a false sense of security is not shown, since for example, a bad mark in confidentiality could be compensated with a good mark in authentication if the marks are combined by means of an arithmetic function. For making this visual, the usage of an pentagon like the triangle in [58] is proposed, where the vertices are the five general vulnerabilities and the profiles are represented by internal lines. It should be noted that in the previous version, we considered an octagon, since we had eight vulnerabilities, as explained in Section 3 At the same time, the visual concept of more area more risk, and the usage of degraded colours (red and green) helps a non-expert consumer to understand the cybersecurity label. Finally, as security is a dynamic concept, the usage of a digital $\mathrm{QR}$ as cybersecurity label is proposed to be updated in case of a new vulnerability is discovered in the product. In this sense, the label (and certificate) is valid unless a recertification is needed or the conditions defined in the security certification process are still valid. For example, a cryptographic algorithm specified in the security profile may become obsolete. Therefore, the communication to the user could be instantaneous through the monitoring process, where the state of the IoT device is periodically or continuously assessed from a security point of view to initiate the recertification process.

In the next section, we described how to apply the described methodology to a specific IoT scenario. 


\section{Applying the proposed methodology in IoT scenarios}

As an example of certification methodology and a way to validate its applicability, we present the results corresponding to one of the ARMOUR scenarios, whose details are shown on Figure 3 In this scenario we label two of the main used IoT protocols: the Constrained Application Protocol (CoAP) [10] and the Datagram Transport Layer Security (DTLS)[11] protocol. CoAP has recently 515 been standardized by the Constrained RESTful Environments (CoRE) working group ${ }^{9}$ as the application protocol for exchanging information between smart objects and, therefore, is specifically designed for devices with small memory and computational resources, such as those expected in IoT networks. In the same context, the DTLS In Constrained Environments (DICE) working group ${ }^{10}$ focuses on supporting the use of DTLS Transport-Layer Security in these environments.

The scenario is mainly motivated by the need to consider suitable mechanisms for security credential management and distribution, so IoT devices can interoperate securely during their operation. Specifically, it is based on the use of CoAP and DTLS with authentication based on preshared key (PSK), so IoT devices can request security credentials (symmetric group keys in this case), which are used later for a secure operation. During the first part of the scenario (key distribution), the group keys are generated from a set of certified attributes used to distinguish the access rights to certain data of different smart objects. Figure 3 provides an overview of the required interactions between the smart object and the Credential Manager (CM), which is responsible for generating and distributing such credentials. The second part of the scenario (secure data sharing) is focused on testing IoT platforms implementing the oneM2M standards ${ }^{11}$ by using a pusbish/subscribe approach. In this case, the smart object publishes some data in the oneM2M platform encrypted with the group key delivered previously, or requests data published by another smart object. In this last case, it also has to decrypt it with the group key delivered in the previous phase. All the publication/subscription process is intended to be done after the establishment of a DTLS secure channel in a similar way that with the CM.

\footnotetext{
${ }^{9}$ https:// datatracker.ietf.org/wg/core/charter/

${ }^{10}$ https://datatracker.ietf.org/wg/dice/about/

${ }^{11}$ http://www.onem2m.org/
} 


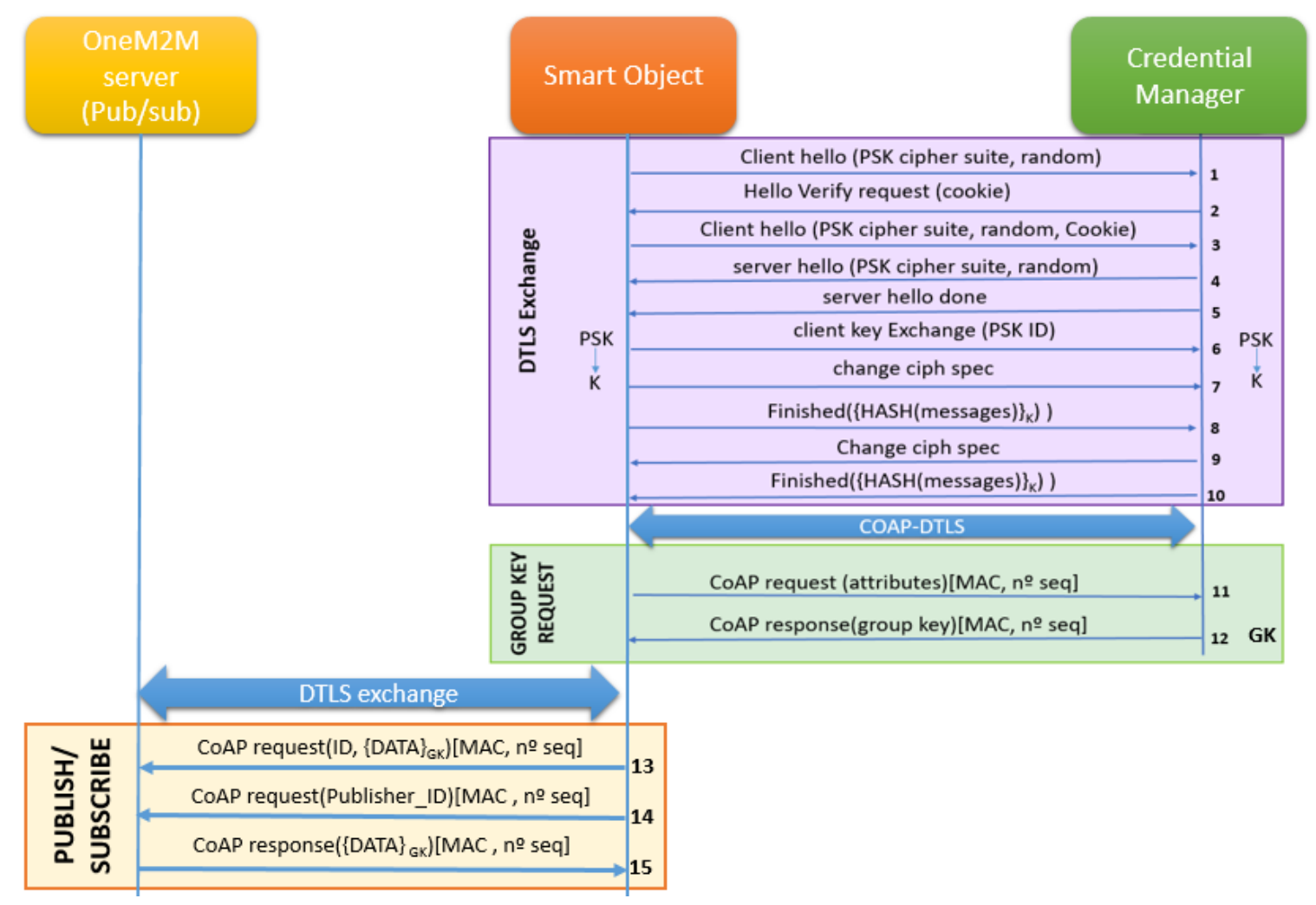

Figure 3: General overview of the considered scenario

For this particular scenario, the TOE is the smart object, a M3 device executed by means of FiT IoT Lab, in addition to the libraries for CoAP (erbium ${ }^{12}$ ) and DTLS (tinydtls v0.5. ${ }^{13}$ ). This also includes the configuration related with the cryptographic algorithms and key lengths and a context [59]. It is worth noting that this is an example and the security level obtained can change depending on context.

\subsection{Risk Identification}

In this first activity, we select which vulnerabilities are applicable to the TOE that is being tested. Firstly, we have searched in the current vulnerability databases (NVD, CWE, CVE, CERT-EU) the discovered applicable vulnerabilities related with the library version. As no vulnerability was found, we directly apply the OneM2M vulnerabilities. In this case, all of them are applicable except the web specific vulnerabilities (scripting and injection).

\footnotetext{
${ }^{12} \mathrm{http}: / /$ people.inf.ethz.ch/mkovatsc/erbium.php

${ }^{13} \mathrm{https}$ // / projects.eclipse.org/projects/iot.tinydtls
} 


\subsection{Test design and implementation}

According to the proposed methodology, once the vulnerabilities are selected, MBT is used to generate the model for the TOE. Furthermore, OCL is used to specify the TOE behaviour and the test purposes. The test design includes the generation of a UML diagram class for the scenario. This way, Figures 4 and 5 show the UML diagram generated for each part of the scenario (key distribution and secure data sharing) being considered.

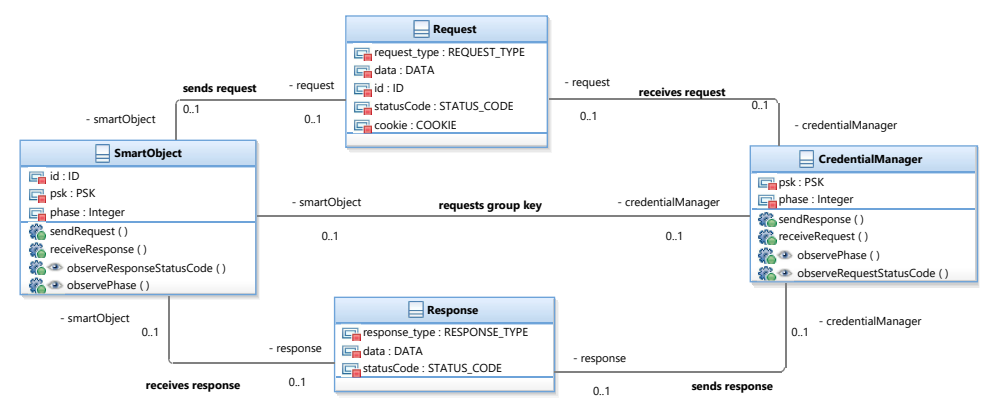

Figure 4: MBT model for the key distribution

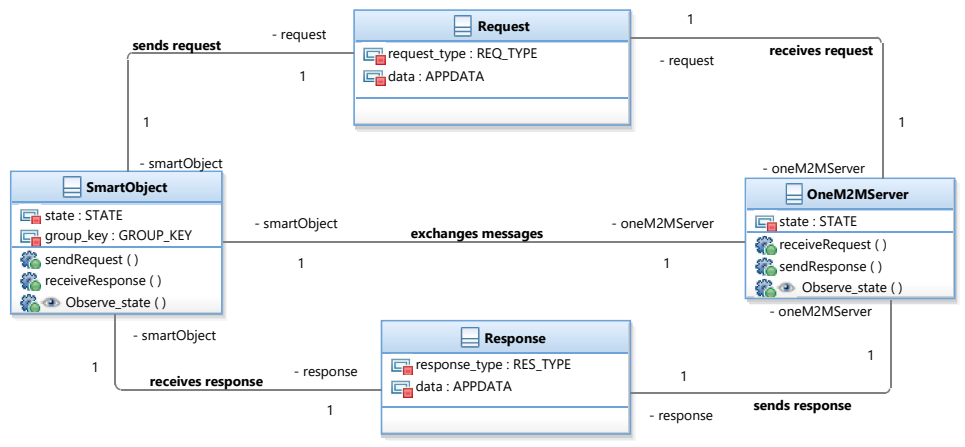

Figure 5: MBT model for the secure data sharing

We have defined four main entities for testing both parts of the scenario: the SmartObject, the server (CredentialManager or OneM2MServer in each case) and the messages, Request and Response. Thus, the SmartObject requests a group key from the CredentialManager (or exchanges messages with the OneM2MServer). Moreover, the CredentialManager receives Requests and sends Responses, whereas the SmartObject sends Requests and receives Responses.

It is worth noting that we have modelled only the operations and fields required for security aspects. In the Requests and Response, we have considered the DATA for modelling a data alteration or an encryption process by using an incorrect key, the STATUS_CODE to analyse the result of an operation, and the REQUEST_TYPE and RESPONSE_TYPE to model the behaviour of the protocol. 
In case of the request, we use the ID to test the lack of authentication and the cookie included in message 2 of Figure 3 (corresponding to DTLS) for lack of availability.

In the first model, we need the ID of the SmartObject, and the PSK shared between both entities to test the lack of authentication. In addition, we use an extra field, PHASE, to control the execution of the protocol (it is related with the message number of Figure 3). In the second model, this field has a similar purpose to the STATE field.

Listing 1: OCL example for the scenario

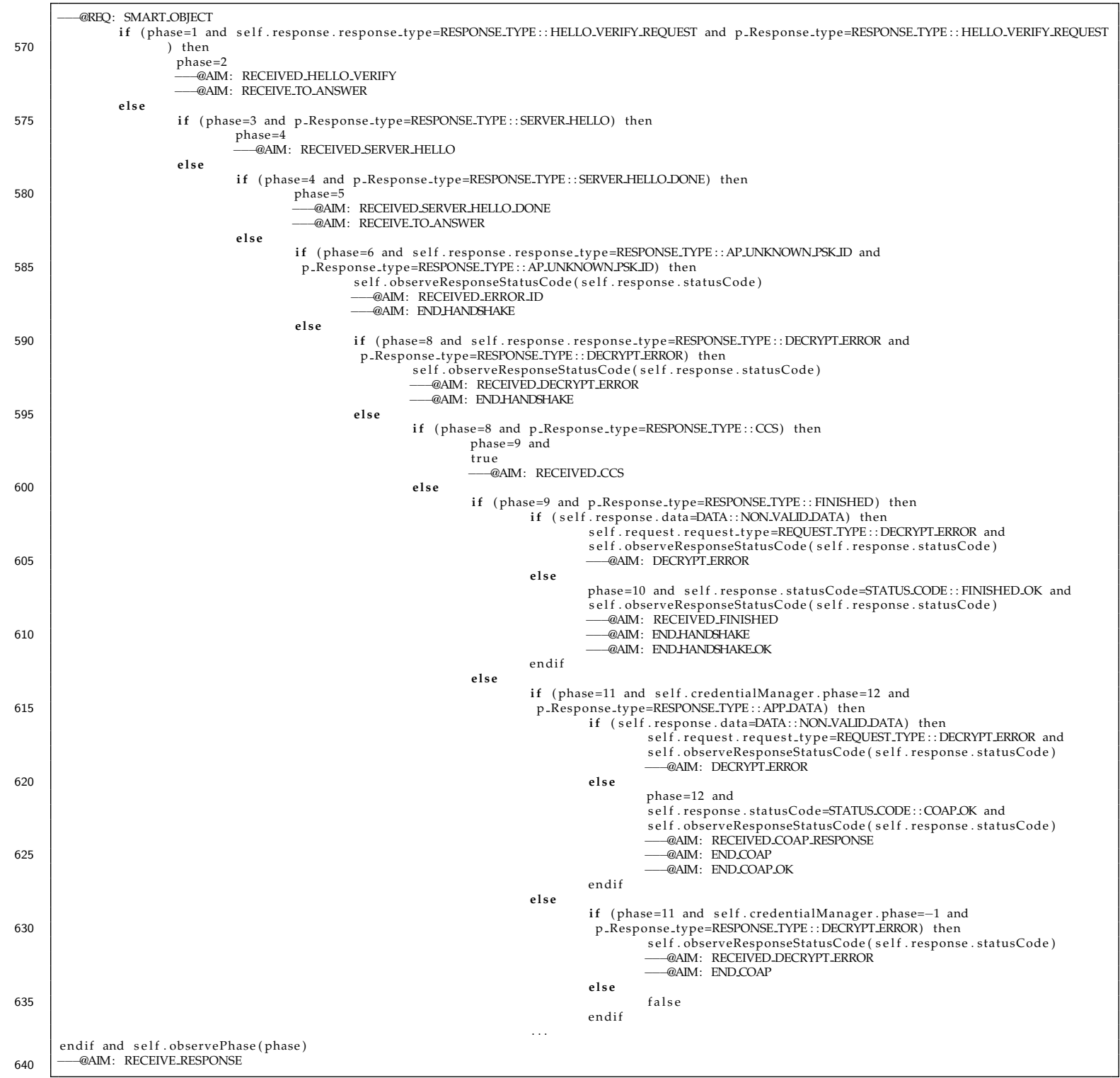

Finally, we have an operation for sending and another for receiving messages and acting conse- 
Table 8: Defined tests for the scenario

\begin{tabular}{|c|c|c|}
\hline Vulnerability & Tests & ID \\
\hline Lack of Confidentiality & $\begin{array}{l}\text { Normal execution of the first part with a sniffer } \\
\text { Normal execution of the second part with a sniffer }\end{array}$ & $\mathrm{C} 1$ \\
\hline \multirow{4}{*}{ Lack of Authentication } & Execution of the scenario with a non valid device ID & \\
\hline & Execution of the scenario with a non valid server PSK & \\
\hline & Execution of the scenario with a non valid device PSK & N3 \\
\hline & Normal execution of the first part with a sniffer & $\mathrm{C} 1$ \\
\hline Lack of Authorization & $\begin{array}{l}\text { Execution of the scenario with a non authorized attributes (and therefore group key) } \\
\text { Normal execution of the second part with a sniffer }\end{array}$ & $\begin{array}{l}\mathrm{Z1} \\
\mathrm{C} 2\end{array}$ \\
\hline \multirow{14}{*}{ Lack of Integrity } & Normal execution of the first part with a sniffer & $\mathrm{C} 1$ \\
\hline & Normal execution of the second part with a sniffer & $\mathrm{C} 2$ \\
\hline & Execution of the scenario with a modified field of Client Hello message (one test for each message field) & $\mathrm{I}^{*}$ \\
\hline & Execution of the scenario with a modified field of Hello Verify Request message (one test for each message field) & $\mathrm{I} 1^{*}$ \\
\hline & Execution of the scenario with a modified field of Client Hello message (one test for each message field) & $\mathrm{I} 2^{*}$ \\
\hline & Execution of the scenario with a modified field of Server Hello message (one test for each message field) & $\mathrm{I}^{*}$ \\
\hline & Execution of the scenario with a modified field of Client Key Exchange message (one test for each message field) & $\mathrm{I}^{*}$ \\
\hline & Execution of the scenario with a modified visible field of Client Finished message (one test for each message field) & $\mathrm{II}^{*}$ \\
\hline & Execution of the scenario with a modified visible field of Server Finished message (one test for each message field) & $\mathrm{I}^{*}$ \\
\hline & Execution of the scenario with a modified visible field of CoAP Key Request (one test for each message field) & $17^{*}$ \\
\hline & Execution of the scenario with a modified visible field of CoAP Key Response (one test for each message field) & $18^{*}$ \\
\hline & Execution of the scenario with a modified visible field of CoAP Request publish data (one test for each message field) & $19^{*}$ \\
\hline & Execution of the scenario with a modified visible field of CoAP Request subscribe data (one test for each message field) & $\mathrm{IA} 0^{*}$ \\
\hline & Execution of the scenario with a modified visible field of CoAP Response data (one test for each message field) & $\mathrm{IA1}^{*}$ \\
\hline Lack of availability & $\begin{array}{l}\text { Execution of the scenario with } X \text { devices sending at the same time a Client Hello, with X from } 1 \text { to crash or to MAX_THRESHOLD. Undefined number of } \\
\text { tests. }\end{array}$ & $\mathrm{A}^{*}$ \\
\hline
\end{tabular}

quently. The operations with an eye icon are observers, used to gather information and determine if the test has been performed correctly or not. As an example of the behaviour specification in OCL, Listing 1 shows the implementation of the receiveResponse() method of the SmartObject in the first model. We use the fields defined before to model the behaviour of the scenario and the security we want to test. In order to define the tests later, we use tags such as RECEIVED_ERRORID or ERROR_HANDSHAKE to specify which state of the scenario we want to achieve.

The tests generated for the specific TOE are described in Table 8. For the lack of authentication, the tests are related to the mutual authentication of both entities, the smart object and the CM, in this case through the usage of a PSK and the PSK ID. We also use information from a packet analyzer related with the key length. In lack of integrity, we perform an integrity test for each field of each message, modifying a random byte but taking into account that the attacker is not able to see the content of the encrypted messages. Finally, the number of tests concerning Lack of availability, in this case DoS attacks, is undetermined, since we increase the number of devices establishing a communication with the CM until it crashes or until a threshold.

As proof of concept, in the next subsection we detail how to generate the tests related to lack of confidentiality. The rest of the tests follow a similar process.

\subsubsection{Lack of Confidentiality}

The test for the lack of confidentiality consists on a normal execution of the entire scenario with a sniffer (i.e., a packet analyzer) as an additional element that is integrated in the execution platform. The process of this test in the first part of the scenario can be described as follows (C1):

1. The Smart Object establishes a secure communication channel through DTLS with the CM.

2. The Smart Object makes a Request to the CM in order to obtain a Group_Key after being established a secure communication channel through DTLS.

3. The CM generates a Group_Key and sends it to the Smart Object in a Response.

4. The Sniffer intercepts the messages exchanged between the Smart Object and the CM in order to gather information about the Group_Key. 
To automatize the generation of this test, we use the tag RECEIVED_COAP_RESPONSE in the test purpose (Figure 6) to indicate that we want to achieve that tag, specified in the operation of the smart object. CertifyIt will be in charge of develop the test to obtain the intermediate valid steps, as shown in Figure 7. For doing this, it analyses all the possibilities of execution taking into account the defined code of operations and it selects a valid sequence that reaches the specified tag.

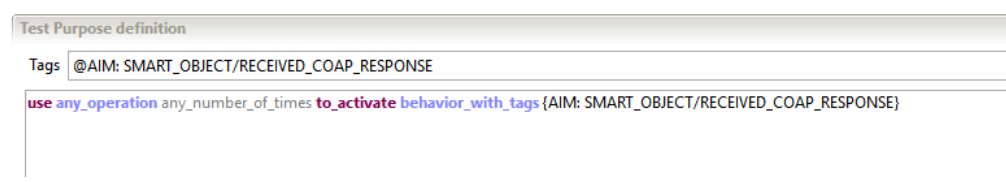

Figure 6: Test purpose definition for lack of confidentiality. C1.

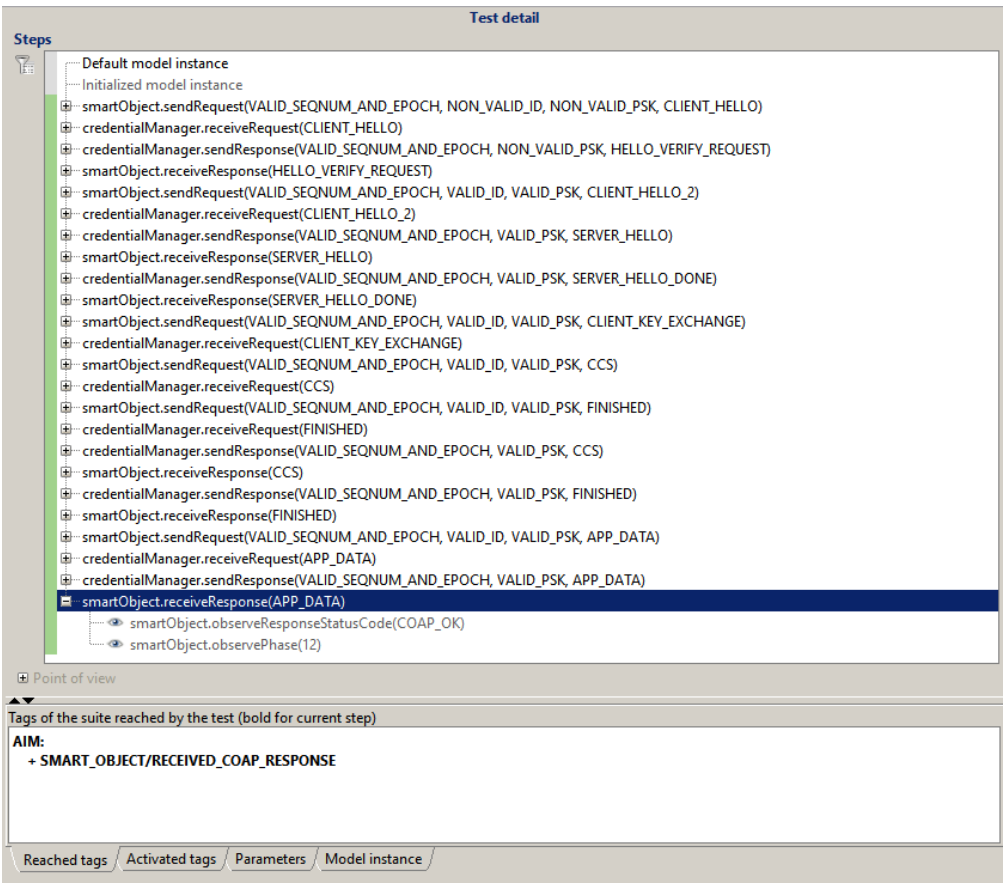

Figure 7: Test generation in CertifyIt for lack of confidentiality. C1.

On other hand the process of the lack of confidentiality test for the second part supposes to establish a secure DTLS connexion with the server (as done previously). As the DTLS establishment test is already generated, we focused on the publication/subscription part in order to have the complete interaction of the scenario. The test follows the next steps (C2):

1. The Smart Object uses the group key obtained from the CM to encrypt some data and it sends it to the OneM2M Server.

2. The Smart Object asks to the OneM2M Server for the data published. 
3. The OneM2M Server sends the encrypted data to it.

4. The Smart Object uses the group key obtained from the CM to decrypt the data.

5. The Sniffer intercepts the messages exchanged between the Smart Object and the OneM2M Server in order to gather information about the exchanged data.

As we want a normal execution of the scenario, we use the tag DECRYPTED_RESPONSE to specify in the test purpose that we want the smart object to receive the data and decrypt it correctly (Figure 8). As before, CertifyIt will check the possible valid intermediate steps to complete the test (Figure 9 ).

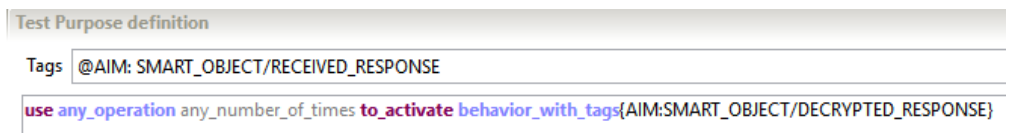

Figure 8: Test purpose definition for lack of confidentiality. C2.

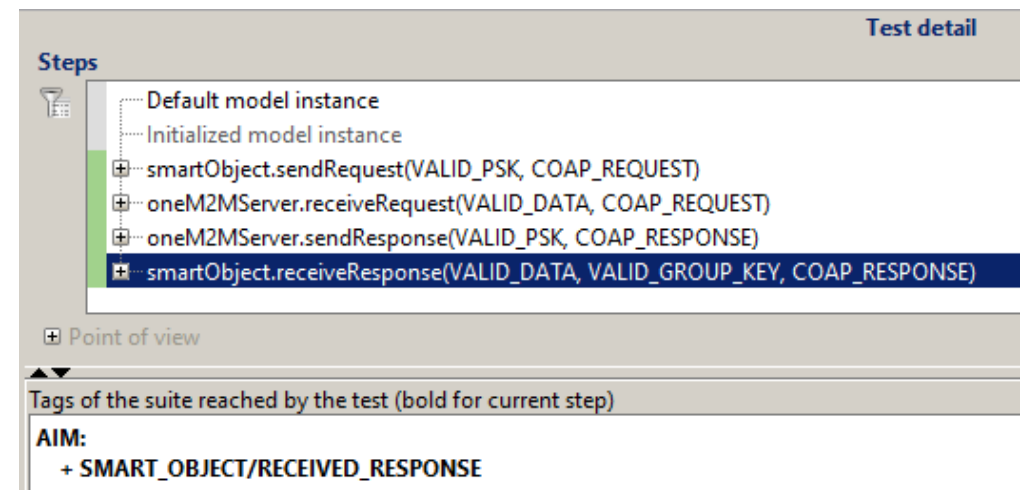

Figure 9: Test generation in CertifyIt for lack of confidentiality. C2.

Both tests are published in TTCN3 language with the help of a generic TTCN3 publisher that is custom built for the ARMOUR project. Figure 10 shows the publisher configuration in CertifyIt. 


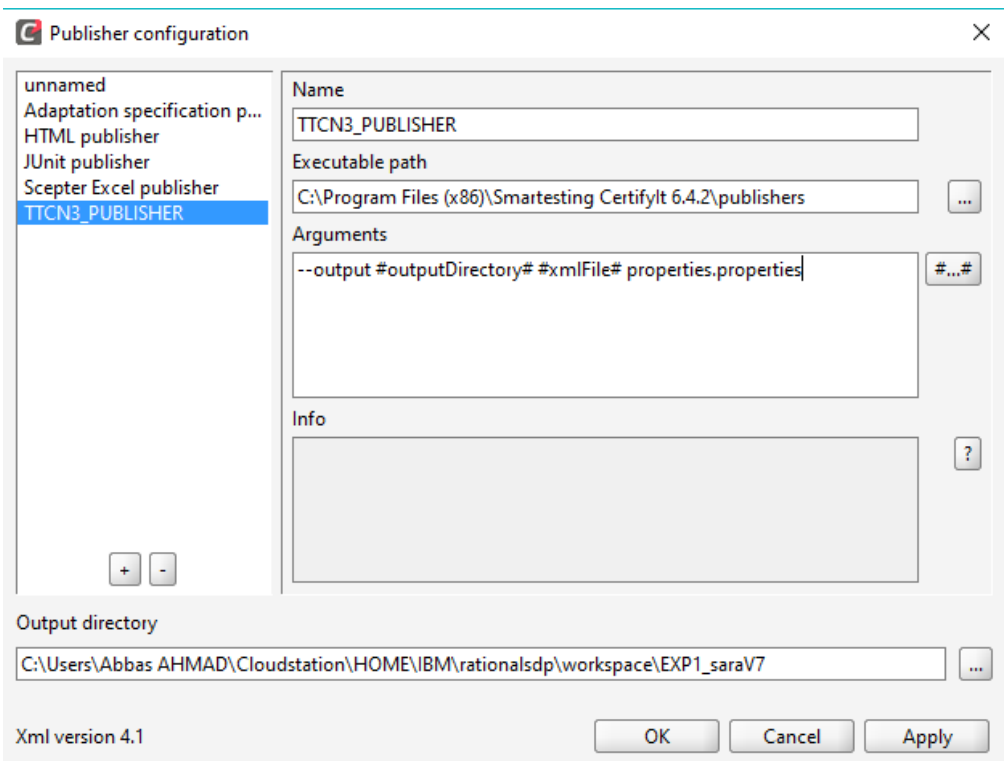

Figure 10: TTCN3 Publisher configuration

690

\section{adapter files. They show respectively the results outcome of the first and second part for lack of confidentiality.}

Listing 2: TTCN-3 code of the first part for lack of confidentiality

695 


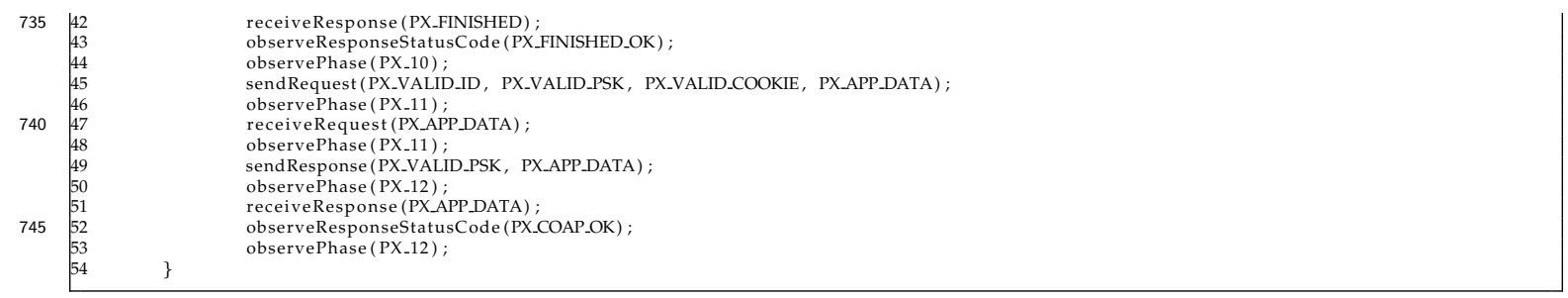

Listing 3: TTCN-3 code of the second part for lack of confidentiality

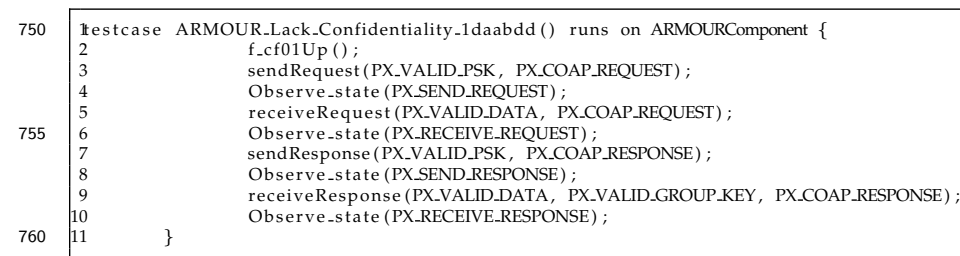

The last step of this phase is to link the generated test case in TTCN-3 with the real implementation of the TOE. CertifyIt generates an interface (adapter) that consists of the functions defined in the TOE model, and the TTCN-3 test itself that makes use of such functions. This interface is then 765 implemented (Experiment controller, EC, in the Figure 11) to make possible the link between the TTCN-3 tests and the real device, in order to allow the execution of each test step.

\subsection{Test environment set up and maintenance}

In this phase, the main work is to set up the testing environment shown in the Figure 11 that in this case is the FIT IoT-LAB platform. As already mentioned, this platform provides valuable features for testing purposes, and it has been used in the scope of the EU ARMOUR project. We make use of two nodes for the SmartObject and a bridge device. These devices (nodes) are based on a STM32 (ARM Cortex M3) micro-controller. They have a 32-bits processing, a new ATMEL radio interface in $2.4 \mathrm{~Hz}$ and a set of sensors. The OS embedded within the M3 nodes is ContikiOS. The M3 node sends and receive data to/from the CM, which is located in a remote virtual machine.

775 This communication is possible due to the border router. As already mentioned, the M3 node uses tinyDTLS v0.5.0 and Erbium libraries for DTLS and CoAP, as Scandium and Californium ${ }^{14}$ for the CM.

The M3 nodes have some communication constraints, they can only transmit data via serial/radio. In order to outcome this constraint, IoT Lab provides an MQTT aggregator which translates the serial message into MQTT topic. TITAN communicates then with the adapter (EC), which is the entry point for the IoT Lab enabling its setup and configuration as well as a bridge to relay devices messages to TITAN coming from the MQTT aggregator.

During this activity, additional entities necessary for the test execution are also set up. This is the case of the packet sniffer that is used to capture and analyse the radio communication traffic. In particular, we make use of the sniffer tool integrated in FIT IoT Lab ${ }^{15}$ to realize this functionality.

\footnotetext{
${ }^{14}$ https://www.eclipse.org/californium/

${ }^{15}$ https://www.iot-lab.info/tutorials/radio-sniffer/
} 
This way, we can capture frames over the air and visualize them in the well known sniffer tool Wireshark 16

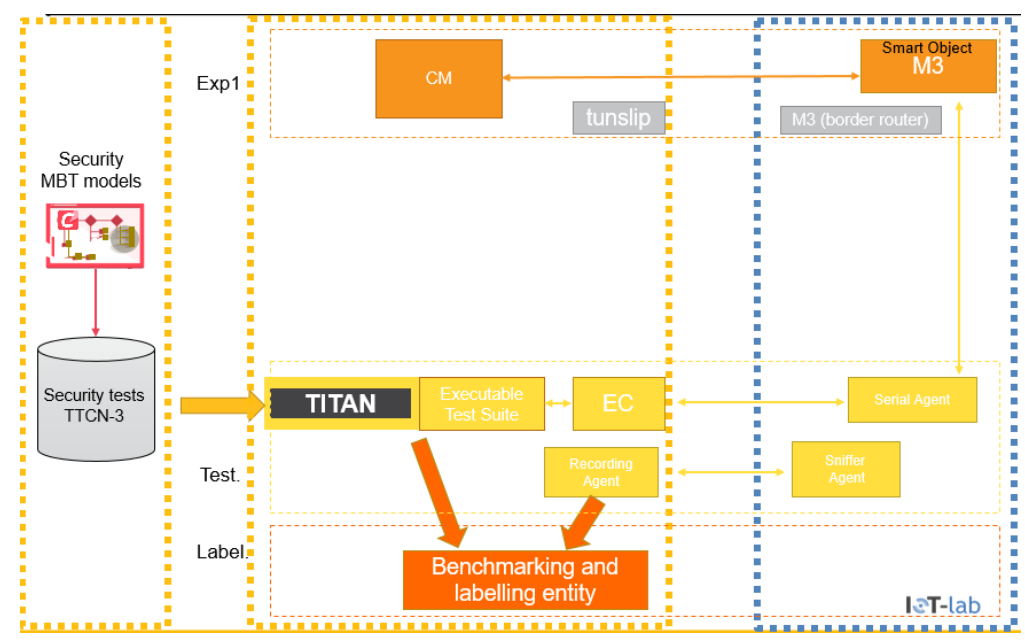

Figure 11: Overall process of testing Lack of confidentiality for the scenario

\subsection{Test execution, analysis and summary}

The corresponding tests are executed in FIT IoT-LAB by means of TITAN that sends the test sequence (sendRequest, sendResponse, etc.) to the TOE, which is executed in FIT IoT-LAB. This communication is possible due to the previous implementation of the adapter. Once the tests are finalized, we obtain the TITAN log file, which helps to know if the execution of the test has been per-formed correctly or not. Listing 4 shows the test ID, the verdict, the test cases executed and the overall status of all of them.

\section{Listing 4: TTCN-3 tests}

$$
15
$$

1.. 1 9:49:22.291554 EXP1_TestPurposeSuite. $t$ tcn:10->ARMOUR_Adapter_HTTP. $t \mathrm{tcn}: 124->$ ARMOUR_Adapter_HTTP. $t \mathrm{tcn}: 110$ Stop timer tc_ac: $120 \mathrm{~s}$

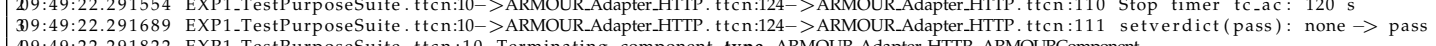

4) $9: 49: 22.291822$ EXP1_TestPurposeSuite. $\operatorname{ttcn}: 10$ Terminating component type ARMOUR_Adapter_HTTP.ARMOURComponent.

5) 9:49:22.291937 EXP1_TestPurposeSuite. ttcn:10 Removing unterminated mapping between port IPL4 and system: IPL4

69:49:22.292008 EXP1_TestPurposeSuite.ttcn:10 IPL4: IPL4asp_-PT_PROVIDER : user_unmap (IPL4): enter

709:49:22.292118 EXP1_TestPurposeSuite.tten:10 IPL4: IPL4asp_-PT_PROVIDER:: user_unmap: There are 1 open connections

89 9:49:22.292247 EXP1_TestPurposeSuite.ttcn:10 IPL4: IPL4asp_.PP_PROVIDER::ConnDel: enter : connId: 1

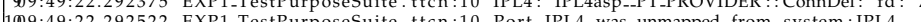

109:49:22.292522 EXP1_TestPurposeSuite.ttcn:10 Port IPL4 was unmapped from system:IPL4.
$109: 49: 22.292633$ EXP1_TestPurposeSuite. $t$ tcn:10 IPL4: IPL4asp_PT_PROVIDER : user_stop : enter

11) 9:49:22.292633 EXP1_TestPurposeSuite. $t$ ttcn:10 IPL4: IPL4asp_-PT_PROVIDE

139:49:22.292818 EXP1_TestPurposeSuite.ttcn:10 Component type ARMOUR_Adapter_HTTP.ARMOURComponent was shut down in testcase

ARMOUR_TP ID 8 - 100 . 770 .

14) 9:49:22.292919 EXP1_TestPurposeSuite ttcn:10 Waiting for PTCs to finish

1509:49:22.293073 EXP1_TestPurposeSuite. $\operatorname{ttcn}: 10$ Setting final verdict of the test case.

169:49:22.293147 EXP1_TestPurposeSuite.tten:10 Local verdict of MTC: pass

1709:49:22.293251 EXP1_TestPurposeSuite. ttcn:10 No PTCs were created.

189:49:22.293369 EXP1_TestPurposeSuite.tten:10 Test case ARMOUR_TP_ID_8_100c770 finished.Verdict: pass

209:49:22.293841 - Test execution summary: 1 test case was executed. Overall verdict:

219:49:22.293937 - Exit was requested from MC. Terminating MTC.

One of the main advantages of FIT IoT Lab is that it allows to execute the scenario at large scale, a useful property to test DoS attacks. In this type of attacks, we can select how many devices we

\footnotetext{
${ }^{16}$ https://www.wireshark.org/
} 
Table 9: Integration of test results with CVSS

\begin{tabular}{llllll}
\hline CVSS Metric & Lack of Confidentiality & Lack of AuthN & Lack of Integrity & Lack of AuthZ & Lack of Availability \\
\hline Attack Vector (AV) & NETWORK $(0.85)$ & NETWORK $(0.85)$ & NETWORK $(0.85)$ & NETWORK $(0.85)$ & NETWORK $(0.85)$ \\
\hline Attack Complexity (AC) & F(TRACE) & F(TRACE) & F(TRACE) & F(TRACE) & F(STD_DEV) \\
\hline Privileges Required (PR) & NONE $(0.85)$ & NONE $(0.85)$ & NONE $(0.85)$ & LOW $(0.68)$ & NONE $(0.85)$ \\
\hline User Interaction (UI) & REQUURED $(0.62)$ & NONE $(0.85)$ & REQUIRED $(0.62)$ & NONE $(0.85)$ & REQUIRED $(0.62)$ \\
\hline AuthZ Scope (S) & UNCHANGED $(0)$ & UNCHANGED $(0)$ & UNCHANGED $(0)$ & CHANGED $(1)$ & UNCHANGED $(0)$ \\
\hline Confidentiality (C) & $\begin{array}{l}\% \text { NON ENCRYPTED } \\
\text { DATA } / 100\end{array}$ & TESTS STATUS & NA $(0)$ & TEST STATUS & NA $(0)$ \\
\hline Integrity (I) & NA $(0)$ & NA $(0)$ & \% BYTES NON PROTECT- & NA (0) & NA (0) \\
\hline Evailability (A) & NA $(0)$ & NA $(0)$ & NA $(0)$ & NA $(0)$ & $\%$ \\
\hline
\end{tabular}

want to use for the test, automating the process of uploading and executing the code, in addition to the execution result. In this case, we gather information related with the number of successful communications to obtain statistical parameters useful for the risk estimation phase, as can be seen in Figure 12 .

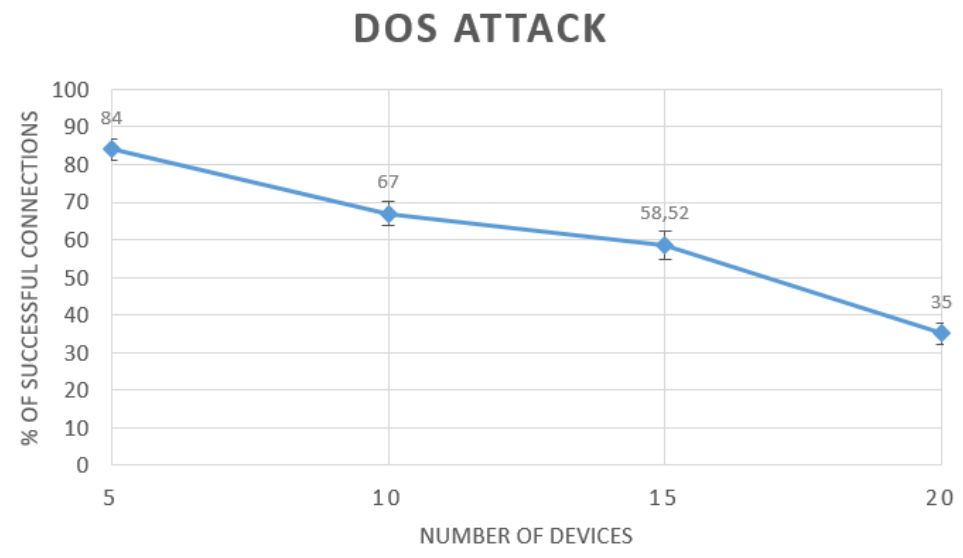

Figure 12: DoS attack summary

Finally, for all the vulnerabilities that have an active sniffer, such as the lack of confidentiality, we also obtain the sniffer trace. This trace will be parsed in order to obtain valuable information to fill the CVSS metrics in a more refined and objective way. This information can include the percentage of non encrypted data, the key length or the cryptographic suite used. In the sniffer trace we can see the general flow of DTLS exchange and some packets labelled with Application data that contain the finished DTLS message encrypted and the delivery of the group key, also encrypted. All the payloads of these packets of data are completely encrypted. However, we can see the version of DTLS, which can be a source of information for a hacker if it is a weak version exploitable due to some bugs. The rest of the packets of the DTLS communication are in clear. We can also see what cipher suite and key length is going to be used.

\subsection{Risk Estimation}

In this activity, the test report is included in the CVSS metrics, by using the results obtained from such report. Table 9 shows this aspects for each vulnerability. 
Table 10: Attack complexity metric

\begin{tabular}{lllr}
\hline $\begin{array}{l}\text { Lack of confidentiality, } \\
\text { Lack of authentication, }\end{array}$ & LOW & $\begin{array}{l}\text { no NIST recommended suite and key } \\
\text { length/MAC }\end{array}$ & 0.77 \\
\cline { 2 - 5 } $\begin{array}{l}\text { Lack of authorization } \\
\text { and Lack of integrity }\end{array}$ & HIGH & $\begin{array}{l}\text { NIST recommended suite and key length- } \\
\text { /MAC }\end{array}$ & 0.44 \\
\hline Lack of availability & LOW & Standard deviation $\leq 10$ & 0.77 \\
\cline { 2 - 4 } & HIGH & Standard deviation $>10$ & 0.44 \\
\hline
\end{tabular}

Table 11: Confidentiality metric for lack of authentication and lack of authorization

\begin{tabular}{lllll}
\hline \multirow{3}{*}{ Confidentiality (C) } & NONE & No authentication/authorization & 0 \\
\cline { 2 - 5 } & LOW & One side authentication/authorization & 0.22 \\
\cline { 2 - 5 } & HIGH & Mutual authentication/authorization & 0.56 \\
\hline
\end{tabular}

As for now, all the vulnerabilities are considered only in the application layer, the attack vector metric from CVSS is always NETWORK. This can be changed in case the vulnerabilities wanted to be considered at different layers. The attack complexity will be taken in general from the sniffer trace (Table 10). It can refer to the key length and cryptographic suite used, MAC length, mechanisms to avoid the attack or the standard deviation of the packet losses for a certain number of simultaneous requests, related with the likelihood that the attack is successful or must be repeated.

Privileges required, user interaction and authorization scope are filled following the CVSS metric description, depending on the vulnerability tested. Finally, the Confidentiality, integrity and availability impact metrics are not applicable or taken from the test status (Table 11) or the sniffer trace (e.g., $\%$ of non encrypted data, $\%$ bytes non integrity protected, mean $\%$ of packet losses for different simultaneous requests, etc.).

Finally we apply the CVSS formulas (Section 3.3) with the metrics values to obtain the risk associated to each vulnerability:

- Lack of confidentiality: We have a percentage of $85 \%$ bytes non encrypted, and a suite/key length recommended by the NIST, Advanced Encryption Standard (AES), with 128 bits key length.

$$
\text { Risk }=\min \{6.42 \cdot(1-(1-0.85)(1-0)(1-0))+8.22 \cdot 0.85 \cdot 0.44 \cdot 0.85 \cdot 0.62,10\}=7.077(7)
$$

- Lack of authentication: We have mutual authentication (both tests passed), and a key length recommended by the NIST (PSK of 128 bits).

$$
\text { Risk }=\min \{6.42 \cdot(1-(1-0)(1-0)(1-0)))+8.22 \cdot 0.85 \cdot 0.44 \cdot 0.85 \cdot 0.85,10\}=0
$$

- Lack of Integrity: We have a percentage of $19 \%$ messages non integrity protected, and a suite/MAC length recommended by NIST protection (AES-CCM-8, 64 bits).

$$
\text { Risk }=\min \{(6.42 \cdot(1-((1-0)(1-0.19)(1-0)))+(8.22 \cdot 0.85 \cdot 0.44 \cdot 0.85 \cdot 0.62), 10\}=2.819
$$

- Lack of authorization: We have one side authorization (one test passed), and a suite/key 
Table 12: Security risk assessment of the experiment

\begin{tabular}{|c|c|c|c|c|c|c|c|}
\hline Vulnerability & Risk & cVss & A & B & C & D & Profiles fulfilled \\
\hline \multirow{4}{*}{$\begin{array}{c}\text { Lack of } \\
\text { confidentiality }\end{array}$} & Low & & $\mathrm{x}$ & $\mathrm{x}$ & $\mathrm{x}$ & $\mathrm{x}$ & \multirow{4}{*}{ C } \\
\hline & Medium & & & $\mathrm{x}$ & $\mathrm{x}$ & $\mathrm{x}$ & \\
\hline & High & $\mathrm{x}$ & & & $\mathrm{x}$ & $\mathrm{x}$ & \\
\hline & Critical & & & & & & \\
\hline \multirow{4}{*}{$\begin{array}{l}\text { Lack of } \\
\text { authentication }\end{array}$} & Low & $\mathrm{x}$ & $\mathrm{x}$ & $\mathrm{x}$ & $x$ & $\mathrm{x}$ & \multirow{4}{*}{ A } \\
\hline & Medium & & & $\mathrm{x}$ & $\mathrm{x}$ & $\mathrm{x}$ & \\
\hline & High & & & & $\mathrm{x}$ & $\mathrm{x}$ & \\
\hline & Critical & & & & & $\mathrm{x}$ & \\
\hline \multirow{4}{*}{ Lack of availability } & Low & & $\mathrm{x}$ & $\mathrm{x}$ & $x$ & $\mathrm{x}$ & \multirow{4}{*}{ B } \\
\hline & Medium & $\mathrm{x}$ & & $\mathrm{x}$ & $\mathrm{x}$ & $\mathrm{x}$ & \\
\hline & High & & & & $\mathrm{x}$ & $\mathrm{x}$ & \\
\hline & Critical & & & & & $\mathrm{x}$ & \\
\hline \multirow{4}{*}{ Lack of integrity } & Low & $\mathrm{x}$ & $\mathrm{x}$ & $\mathrm{x}$ & $x$ & $\mathrm{x}$ & \multirow{4}{*}{ A } \\
\hline & Medium & & & $x$ & $\mathrm{x}$ & $\mathrm{x}$ & \\
\hline & High & & & & $\mathrm{x}$ & $\mathrm{x}$ & \\
\hline & Critical & & & & & $\mathrm{x}$ & \\
\hline \multirow{4}{*}{$\begin{array}{c}\text { Lack of } \\
\text { authorization }\end{array}$} & Low & $\mathrm{x}$ & $\mathrm{x}$ & $\mathrm{x}$ & $x$ & $\mathrm{x}$ & \multirow{4}{*}{ A } \\
\hline & Medium & & & $\mathrm{x}$ & $\mathrm{x}$ & $\mathrm{x}$ & \\
\hline & High & & & & $\mathrm{x}$ & $\mathrm{x}$ & \\
\hline & Critical & & & & & $\mathrm{x}$ & \\
\hline
\end{tabular}

length recommended by the NIST (group key of 128 bits and AES).

$$
\begin{gathered}
I S C=1-((1-0)(1-0)(1-0))=0 \\
E_{S}=8.22 \cdot 0.85 \cdot 0.44 \cdot 0.68 \cdot 0.62=3.1 \\
\text { Risk }=\min \left\{1.08 \cdot\left(7.52 \cdot(I S C-0.029)-3.25 \cdot(I S C-0.02)^{15}\right)+E_{S}, 10\right\}=0
\end{gathered}
$$

- Lack of availability: We have a percentage of $61.13 \%$ of packet losses and a standard deviation of 12.61 .

$$
\begin{gathered}
I S C=1-((1-0)(1-0)(1-0.61))=0.61 \\
E_{S}=8.22 \cdot 0.85 \cdot 0.44 \cdot 0.85 \cdot 0.85=2.22
\end{gathered}
$$

$$
\text { Risk }=\min \left\{1.08 \cdot\left(7.52 \cdot(\text { ISC }-0.029)-3.25 \cdot(\text { ISC }-0.02)^{15}\right)+E_{S}, 10\right\}=6.971
$$

\subsection{Risk Evaluation}

In this activity, we perform a mapping between the scores calculated before and the CVSS risk levels in order to compare them with the available profiles.

As we can see in Table 12. CoAPs fulfils for lack of confidentiality the profiles C and D, so as we choose always the highest profile, the label assigned to this vulnerability will be $\mathrm{C}$. In this case, the lack of confidentiality is high due to the DTLS handshake that is in clear until it finishes and therefore, the channel is protected.

\subsection{Security Labelling}

The obtained profile in each vulnerability generates the final label for the example experiment (CoAPs). The result of the label is presented to the user in a multidimensional label like in Figure 13 As already mentioned, following ECSO recommendations, this label includes a QR. This aspect 


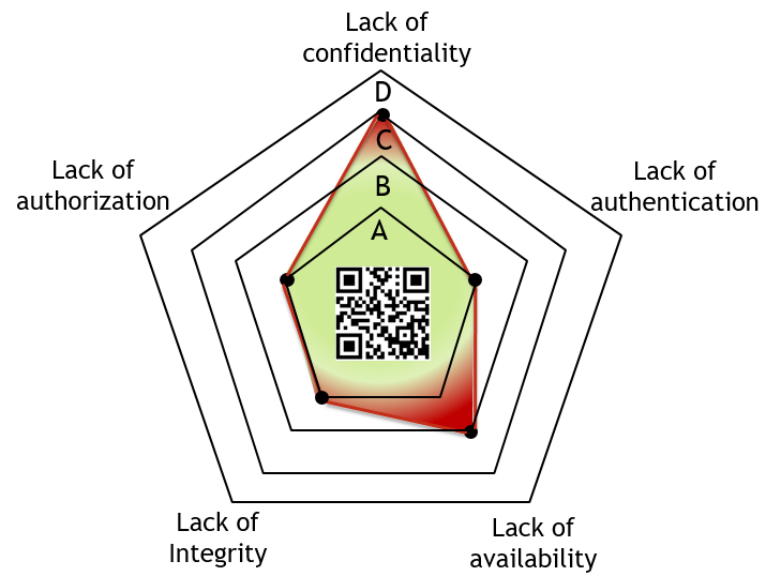

Figure 13: Multidimensional label obtained by the example experiment in one context

is intended to provide additional information about the certification process that can be employed to get a more precise view of the security level associated with a specific TOE. Furthermore, the QR is used to maintain the label updated, so any change in the security level of the TOE can be efficiently reflected.

As an example of the implementation of the process, Figure 14 shows a Java application that automatizes the security test analysis and risk assessment processes showing the final profile obtained for each vulnerability. The Figure also shows the details about the lack of confidentiality risk calculation. In this case, the program analyses the metrics obtained from the TITAN log (right) and the sniffer trace parsed previously with a script (left) to obtain the CVSS risk (7.08), the equivalent risk interval (High) and the profile obtained for lack of confidentiality (C). We can see that the used metrics in this vulnerability are the overall status of the TITAN tests $(2 / 2$ have passed), the percentage of non encrypted data (85\%), the cryptographic suite (TLS with PSK) and the key length (128 bits).

\section{Discussion}

The proposed methodology is intended to provide a basis to build a cybersecurity framework, solving some of the main challenges described in section 2

In this sense, the proposal takes into account the whole life cycle of an IoT device. The certification process is not exclusive of the manufacturing phase, but it includes monitoring the devices during its life cycle and recertificate it in case there is a change on the security level offered or needed. The inclusion of the domain in the cybersecurity label and therefore the applicability of the proposal to all of them, copes with the heterogeneity of existing schemes, providing a unique and integrated scheme for security certification. The definition of a specific domain can be based on the recent proposal in [59], which defines Consumer (domestic), Enterprise, Industrial, Medical, Automotive, Public agency and Critical National Infrastructure domains.

The automation of the testing process by means of technologies such as MBT, CertifyIt and TITAN to design, generate and execute the security tests, produces an easy, fast and cost effective recertification process, coping with one of the major challenges of a cybersecurity certification 


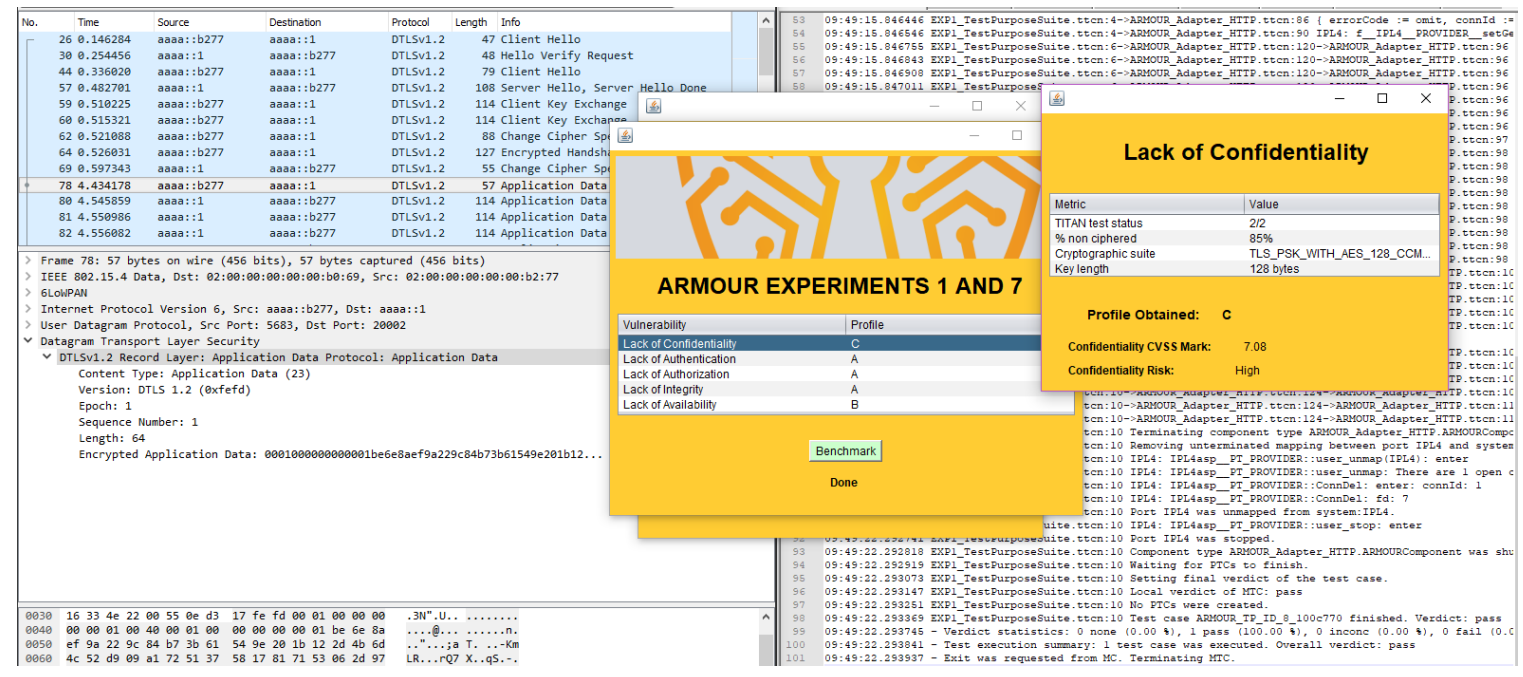

Figure 14: Demo for labelling the experiment

Table 13: Challenges addressed by the proposed approach

\begin{tabular}{ll}
\hline Challenge Addressed & Solution \\
\hline Heterogeneity of existing schemes & Simple and fast once the automation process is established. It is based on well-known standards. \\
\hline Burden of existing schemes & Simple and fast once the automation process is established. It is based on well-known standards. \\
\hline Standardization & It is based on well-known standards. \\
\hline Dynamism & Fast recertification process due to testing process automation. \\
\hline Consider the device life cycle & The certification process is active during the whole life cycle. \\
\hline Scalability & Fast recertification process due to testing process automation. \\
\hline Cybersecurity label specification & $\begin{array}{l}\text { Usage of a digital QR-code in the cybersecurity label to keep it updated, visual (more area, more risk) and useful (spider chart with the general } \\
\text { vulnerabilities). }\end{array}$ \\
\hline $\begin{array}{l}\text { Influence of the context or operating envi- } \\
\text { ronment }\end{array}$ & Labelling for all the contexts in the manufacturing phase \\
\hline
\end{tabular}

framework: the dynamism of security. One of the reasons to perform a recertification is an update, patch or new vulnerability discovery affecting the device. In this case, the monitoring process should detect this change and assess if a recertification process is required. However, a change on the security level can be also triggered due to a re-ownership that changes the configuration or the domain. The scalability is also addressed in the sense that the certification scheme is intended to be performed in a fast and cost effective manner, in order to cope with the high amount of devices that eed to be certified.

Furthermore, we have taken advantage of the standardized methodology of ETSI, in order to avoid reinventing the wheel, and to promote its acceptance. In addition, the design of the cybersecurity label includes the recommendations proposed by organizations such as ENISA or AIOTI, coping with the tradeoff between simplicity (with the subjectivity associated) and technical information being provided. In addition, the cybersecurity label is to be updated with the current certification process and security level, thanks to the usage of a digital QR code. As already mentioned, this label is intended to be part of a security certificate that embraces other aspects related to the certification process

Finally, a brief summary of the challenges addressed is shown in Table 13

It should be noted that other challenges will be considered as part of our future work in this area. In this sense, a key point is the certification of multiple layers of the protocols stack. So far, 
in the context of the ARMOUR project, some of the experiments being considered are focused on application and transport layers, but we will study how it could be extended and combined with certification at internet and link layer. In this sense, we are considering the usage of the power mean function for aggregating metrics, but the main objective is to continue having only one label for device and context in order to make it easier for the consumer. The physical layer should be studied, since it is difficult to anticipate the physical security conditions that the device will have, before it is deployed. Currently, there are existing approaches to aggregate risk marks from several layers such as the solution presented in RASEN project [50] by means of the usage of vignettes in CWSS. However, it should be analyzed if this proposal could be extended to IoT scenarios. Although it has not been considered, lack of privacy is a challenging aspect that should be taken into account as a main vulnerability, and that should be part of the cybersecurity certificate, providing a clear level of the privacy provided and the associated risks. In this sense, the inclusion of Privacy Impact Assessment (PIA) process could help to define such aspects and related privacy requirements to be part of the certification framework. Finally, the creation of an IoT vulnerability database is out of the scope of the cybersecurity certification framework. In this sense, examples such as the NVD in the U.S can be considered for the definition of such database in the context of IoT in Europe.

\section{Conclusions}

Nowadays, the IoT ecosystem demands for large-scale deployments, where devices must provide a high level of security, in order to cover typical vulnerabilities increasing the acceptance IoT scenarios. In this sense, a cybersecurity certification framework for IoT can help to support the development and deployment of trusted IoT systems, empowering testers and consumers with the ability to assess security solutions for large-scale IoT deployments. The development of this framework has to cope with several challenges, such as the heterogeneity of the devices and existing schemes, the design of the cybersecurity label and the absence of a dedicated IoT vulnerability database. In this sense, one of the most important challenges is the dynamic nature of security, making necessary an efficient and cost-effective recertification process. Towards this end, there is a real need to consider a systematic and automated methodology that enables scalable testing approaches for security aspects in IoT. In this direction, this work aimed to describe a cybersecurity framework proposal based on ETSI and ISO standards, in order to cope with some of the challenges associated with the realization of this framework. In particular, the proposal includes the automation of the whole process by means of technologies such as MBT, CertifyIt and TITAN to design, generate and execute the security tests, in such a way that the recertification process can be made in an easy way. The applicability of this proposal has been tested through its application to a concrete TOE, taking into account specific IoT technologies in the context of the H2020 ARMOUR project. While the definition of a cybersecurity certification framework still needs coordinated efforts from stakeholders and regulatory bodies, the proposed approach is intended to serve as a cornerstone to define a more consistent and standardized approach. In this sense, this methodology is expected to evolve around the ECSO and ENISA guidelines in the coming years.

Future work will be centred on addressing the rest of the challenges discussed in Section 2. One of this challenges is the aggregation of multiple components and layers keeping only one label for device and context in order to make it easier for the consumer. In this sense, the power mean function and the concept of vignettes of CWSS applied to this purpose in RASEN project are being considered to perform the aggregation. Lack of privacy is intended to be added as a new general vulnerability and studied to obtain objective and useful metrics for the risk assessment phase. In 
this sense, the inclusion of Privacy Impact Assessment (PIA) process could help to define such aspects and related privacy requirements to be part of the certification framework.

\section{References} URL http://nvlpubs.nist.gov/nistpubs/Legacy/SP/nistspecialpublication800-137 pdf challenges, Wireless Networks 20 (8) (2014) 2481-2501.

[2] Q. M. Ashraf, M. H. Habaebi, Autonomics chemes for threat mitigation in internet of things, Journal of Network and Computer Applications (49) (2015) 112-127.

[3] C. Kolias, G. Kambourakis, A. Stavrou, J. Voas, ddos in the iot: Mirai and other botnets, IEEE Computer 50 (2017) 80-84.

[4] ENISA, On the security, privacy and usability of online seals. an overview. (Dec. 2013).

[5] ECSO,WG 1: Standardization certification labeling and supply chain management URL https://www.ecs-org.eu/documents/publications/5a3112ec2c891.pdf

[6] DIGITALEUROPE, Digitaleuropes views on cybersecurity certification and labelling schemes (Mar. 2017).

[7] ETSI, Methods for testing \& specification; risk-based security assessment and testing methodologies (2015).

[8] F. Bouquet, C. Grandpierre, B. Legeard, F. Peureux, N. Vacelet, M. Utting, A subset of precise UML for model-based testing, in: 3rd int. Workshop on Advances in Model Based Testing, 2007, pp. 95-104.

[9] FIRST, Common vulnerabilities scoring system (CVSS) (2014). URL https://www.first.org/cvss

[10] Z. Shelby, K. Hartke, C. Bormann, The constrained application protocol (CoAP) (2014). URL https://tools.ietf.org/html/rfc7252

[11] E. Rescorla, N. Modadugu, Datagram transport layer security version 1.2. RFC 6347 (Jan. 2012). URL https://tools.ietf.org/html/rfc6347

[12] S. N. Matheu-Garca, J. L. Hernndez-Ramos, A. F. Skarmeta, Test-based risk assessment and security certification proposal for the internet of things, in: IEEE 4rd World Forum on Internet of Things, 2018.

[13] NIST, Fips 200 (2006).

[14] C. on National Security Systems, Glossary (2015). URL https://cryptosmith.files.wordpress.com/2015/08/glossary-2015-cnss.pdf

[15] NIST, Special publication 800-137 (2011).

pdf

[1] Q. Jing, A. Vasilakos, J. Wan, J. Lu, D. Qiu, Security of the internet of things: perspectives and 
[16] International Organization for Standardization, Iso/iec 31000 - risk management URL https://www .iso.org/iso-31000-risk-management.html

[17] NIST, Special publication 800-55 revision 1 (2008). URL http://nvlpubs .nist.gov/nistpubs/Legacy/SP/nistspecialpublication800-55r1. pdf

[18] FIPS, Security requirements for cryptographic modules (2001). URL https://nvlpubs.nist.gov/nistpubs/FIPS/NIST.FIPS.140-2.pdf

[19] International Organization for Standardization, Iso/iec 27000. URL https://www.itgovernance.co.uk/iso27000-family

[20] CCRA, Common criteria URL http://www. commoncriteriaportal.org

[21] F. Keblawi, D. Sullivan, Applying the common criteria in systems engineering, IEEE Security Privacy 4 (2) (2006) 50-55.

[22] S. P. Kaluvuri, M. Bezzi, Y. Roudier, A quantitative analysis of common criteria certification practice, in: International Conference on Trust, Privacy and Security in Digital Business, 2014.

[23] J. Hearn, Does the common criteria paradigm have a future?, EEE Security \& Privacy 2 (1) (2004) 64-65.

[24] Commercial product assurance (CPA) URL/https://www.ncsc.gov.uk/scheme/commercial-product-assurance-cpa

[25] UL-2900 URL https://standardscatalog.ul.com/standards/en/outline_2900-1_2

[26] ANSSI, Certification de scurit de premier niveau (CSPN) (2008). URL https://www.ssi.gouv.fr/administration/produits-certifies/cspn/

[27] Unabhngiges Landeszentrum fr Datenschutz Schleswig-Holstein, ULD datenschutz-gtesiegel URL https://www .datenschutzzentrum.de/guetesiegel/

[28] ICSA, Internet of things (IoT) security testing framework (2016).

[29] IoT Security Fundation, Iot security compliance framework (2016).

[30] O. T. Alliance, IoT security \& privacy trust framework v2.5 (2017). URL https://otalliance.org/system/files/files/initiative/documents/iot_trust_ framework6-22.pdf

[31] R. Anderson, S. Fuloria, Certification and evaluation: A security economics perspective, in: IEEE (Ed.), Emerging Technologies \& Factory Automation, ETFA, 2009, pp. 1-7.

[32] S. Murdoch, M. Bond, R. Anderson, How certification systems fail: Lessons from the ware report, IEEE Security \& Privacy 10 (6) (2012) 40-44.

[33] Kaluvuri, S. Paul, M. Bezzi, Y. Roudier, A quantitative analysis of common criteria certification practice, Trust, Privacy, and Security in Digital Business (2014) 132-143. 
[34] AIOTI, Report on workshop on security and privacy in the hyper-connected world (2016).

[35] ECSO, A meta-scheme approach v1.0 (2017).

[36] E. Commission, Proposal for a REGULATION OF THE EUROPEAN PARLIAMENT AND OF THE COUNCIL on ENISA, the "EU Cybersecurity Agency", and repealing Regulation (EU) 526/2013, and on Information and Communication Technology cybersecurity certification ("Cybersecurity Act") (2017).

[37] Microsoft, DREAD scheme.

URL https://msdn.microsoft.com/en-us/library/ff648644.aspx

[38] J. R. Nurse, S. Creese, D. D. Roure, Security risk assessment in internet of things systems, IEEE Computer Society, IT Pro.

[39] R. A. Caralli, J. F. Stevens, L. R. Young, W. R. Wilson, Introducing OCTAVE allegro: Improving the information security risk assessment process, Tech. rep., CERT (2007).

a URL http://resources.sei.cmu.edu/asset_files/TechnicalReport/2007_005_001_ 14885.pdf

[40] MITRE, Common weakness scoring system (CWSS) (2014).

URL https://cwe.mitre.org/cwss/cwss_v1.0.1.html

[41] M. Felderer, M. Bchler, M. Johns, A. D. Brucker, R. Breu, A. Pretschner, Security Testing: A Survey, Elsevier, 2016, Ch. 1, pp. 1-51.

[42] B. Arkin, S. Stender, G. McGraw, Software penetration testing, IEEE Security \& Privacy 3 (1) (2005) 84-87.

[43] M. Felderer, B. Agreiter, P. Zech, R. Breu, A classification for model-based security testing, in: Third International Conference on Advances in System Testing and Validation Lifecycle, 2011.

[44] P. Godefroid, M. Y. Levin, D. Molnar, Sage: whitebox fuzzing for security testing, Communications of the ACM 55 (3) (2012) 40-44.

[45] S. Yoo, M. Harman, Regression testing minimization, selection and prioritization: a survey, Software Testing, Verification and Reliability 22 (2) (2012) 67-120.

[46] B. Regnell, P. Runeson, C. Wohlin, Towards integration of use case modelling and usage-based testing, Journal of Systems and Software 50 (2) (2000) 117-130.

[47] M. A. Schneider, S. Herbold, M.-F. Wendland, J. Grabowski, Improving security testing with usage-based fuzz testing, in: Risk Assessment and Risk-Driven Testing, 2015.

[48] W. Li, F. L. Gall, N. Spaseski, A survey on model-based testing tools for test case generation, in: The 4th International Conference on Tools and Methods of Program Analysis, 2017.

[49] M. Schneider, J. Grossmann, I. Schieferdecker, A. Pietschker, Online model-based behavioral fuzzing, in: IEEE Sixth International Conference on Software Testing, Verification and Validation Workshops, 2013. 
[50] RASEN project, D3.2.3. techniques for compositional test-based security risk assessment v.3 (2015).

[51] J. Gromann, F. Seehusen, Combining security risk assessment and security testing based on standards, in: 3rd RISK Workshop, 2015.

[52] ARMOUR project, D1.1: ARMOUR experiments and requirements (2016).

[53] K. Moore, R. Barnes, H. Tschofenig, Best current practices for securing internet of things (IoT) devices (2016).

[54] M. Abomhara, G. M. Kien, Cyber security and the internet of things: Vulnerabilities, threats, intruders and attacks, Journal of Cyber Security 4 (2015) 65-88.

[55] F. A. Alaba, M. Othman, I. A. T. Hashem, F. Alotaibi, Internet of things security: A survey,

1. Journal of Network and Computer Applications 88 (2017) 10-28. doi:10.1016/j.jnca. 2017. 04.002.

[56] E. Commission, Directive 2010/30/EU on the indication by labelling and standard product information of the consumption of energy and other resources by energy-related products (2010).

URL http://eur-lex.europa.eu/legal-content/EN/TXT/?uri=CELEX : 32010L0030

[57] G. Bernabeu, E. Jaffuel, B. Legeard, F. Peureux, MBT for global platform compliance testing: Experience report and lessons learned, in: 25th IEEE International Symposium on Software Reliability Engineering Workshops, 2014.

[58] E. Commission, Best available techniques reference document for the cyber-security and privacy of the 10 minimum functional requirements of the smart metering systems, Smart-Grid Task Force Stakeholder Foru (2016).

[59] I. S. Fundation, Iot security compliance framework (2016). IoT-Security-Compliance-Framework.pdf 